\title{
INFINITE LINEAR SYSTEMS WITH HOMOGENEOUS KERNEL OF DEGREE - 1
}

\author{
T. M. CHERRY
}

(received 5 July 1964)

\section{Introduction}

1.1. The main concern of this paper is with the solution of infinite linear systems

$$
x_{m}+\sum_{n=N}^{\infty} k(m, n) x_{n}=g_{m}, \quad m=N, N+1, \cdots, N \geqq 1
$$

in which the kernel $k$ is a continuous function of real positive variables $m, n$ which is homogeneous with degree -1 , so that

$$
k(m, n)=m^{-1} k(1, z) \quad z=n / m .
$$

If $k$ is a rational algebraic function it is supposed further that the continuity extends up to the axes $m=0, n>0$ and $n=0, m>0$; the possibly additional restriction when $k$ is not rational is discussed in $\S 1.2$.

If the degree of $k(m, n)$ were $-d$ with $d>1, \sum \sum_{m, n=1}^{\infty}|k(m, n)|^{2}$ would converge, and Hilbert's theory based on completely continuous bilinear forms over Hilbert space [1] would be applicable; the system would be equivalent to an integral equation of Fredholm type. It will be shown that, when $d=1$, the system behaves like a singular integral equation with Cauchy-type kernel; in typical cases, where the sequence $\left\{g_{m}\right\}$ is arbitrary apart from convergence conditions, (1.1) can have several solutions $\left\{x_{n}\right\}$, with $\sum k(m, n) x_{n}$ converging at different rates for the different solutions.

The general theory is given in $\S \S 2-6$, with a statement of its results in $\S \S 4.9,6.1$ and 6.4. $\S 7$ discusses asymptotic formulae for the solutions and procedures for their approximate calculation. With trivial modification the theory covers the integral equation

$$
x(m)+\int_{N}^{\infty} k(m, n) x(n) d n=g(m), \quad \quad N>0
$$

analogous to (1.1); this presents the simpler problem, and explicit formulae for its solution are obtained in $\S 4.10$.

In $\S 8$ there is a summary consideration of systems that are more general than but sufficiently 'close' to (1.1). With $k(m, n)$ as in (1.1), 
the new systems have kernels of the forms (i) $a_{m} k(m, n)$ with $\left(a_{m}\right)$ a bounded set of constants, (ii) $k(m, n)\left(1+a_{m}\right)\left(1+b_{n}\right)$, (iii) $k(m, n)\left(1+d_{m n}\right)$ with $a_{m}, b_{n}, d_{m n}$ suitably small.

1.2. Hypotheses. Our theory is based on the simple remark that, if the kernel is expressible in the form

$$
k(m, n)=\int f(m, s) g(n, s) d s
$$

in which the variables $m, n$ are separated in the integrand, then (1.1) expresses $x_{m}$ in terms of the auxiliary variable $\sum x_{n} g(n, s)$, and an integral equation for this auxiliary is immediately derivable. More than one representation of the form (1.3) is possible; the fruitful one is

$$
k(m, n)=\frac{1}{2 \pi i} \int_{c-i \infty}^{c+i \infty} K(s) m^{s-1} n^{-s} d s, \quad 0<c<1
$$

which by (1.2) is a consequence of the Mellin-type representation of the kernel

$$
k(1, z)=\frac{1}{2 \pi i} \int_{c-i \infty}^{c+i \infty} K(s) z^{-s} d s, \quad 0<c<1 .
$$

To validate the theory $K(s)$ must be suitably restricted, and the working hypothesis which supplements (1.2) is

for $z$ real positive $k(1, z)$ is expressible in the form (1.5) where, with the notation

$$
s=\sigma+i t
$$

$K(s)$ is regular in the band ${ }^{1} 0<\sigma<1$, and for $|t|$ sufficiently large is regular in $-1<\sigma<2$ with

$$
K(s)=O\left(e^{-\lambda|t|}\right)
$$

uniformly in $\sigma$, for some positive constant $\lambda$.

Under the assumption of Mellin's theorem, $K(s)$ in (1.5) is determined by analytic continuation from

$$
K(s)=\int_{0}^{\infty} k(1, z) z^{a-1} d z,
$$

whence the satisfaction of the hypothesis just stated may be investigated. For example, it is sufficient that $k(1, z)$ be an analytic function regular at $z=0$ and in the sector $|\arg z| \leqq \delta$, and be single valued and $O\left(z^{-1}\right)$ for $z \sim \infty$; for then the continuation of (1.8) is

$$
K(s)=(2 i)^{-1} \operatorname{cosec} \pi s \int_{C} k(1, z)(-z)^{s-1} d z
$$

1 The term 'strip' is reserved for more particular use; see $\S 2.3$. 
where $C$ is a closed contour consisting of segments of the rays $\arg z= \pm \delta$ and circular arcs near $z=0, \infty$ on which $|\arg (-z)| \leqq \pi-\delta$. Hence $K(s)$ $\sin \pi s$ is an integral function and there is an inequality of the form

$$
|K(s)| \leqq A|\operatorname{cosec} \pi s| \exp (C|\sigma-1|+(\pi-\delta)|t|) ;
$$

so (1.7) is satisfied not merely for $-1<\sigma<2$ but for $|\sigma / t|$ sufficiently small. This hypothesis covers all rational $k(m, n)$ which are continuous for $m \geqq 0, n>0$ and $m>0, n \geqq 0$ and homogeneous with degree - ; such a function gives the form

$$
k(1, z)=\Sigma \frac{A}{(z+a)^{r}},
$$

a finite sum of partial fractions in which each term has $a \neq 0$ and $|\arg a|<\pi$, and (1.9) gives

$$
K(s)=\pi \operatorname{cosec} \pi s \sum A\left(\begin{array}{c}
r-1-s \\
r-1
\end{array}\right) a^{s-r} .
$$

The satisfaction of (1.5) by these forms may be verified by an elementary calculation, without appeal to the general Mellin theorem.

In (1.9) it is not required that $k(1, z)$ be globally single-valued; for example we can have $k(1, z)=\left(1+2 a z \cos \theta+a^{2} z^{2}\right)^{-\frac{1}{2}}$ with $a>0$ and $-\pi<\theta<\pi$, which gives $K(s)=\pi a^{-s} \operatorname{cosec} \pi s \mathrm{P}_{s-1}(\cos \theta)$.

Another class of suitable kernels consists of meromorphic ones representable by (1.10) with an infinite series on the right, provided that (i) each term has $a \neq 0$, (ii) the set $(\pi-|\arg a|)$ has a positive lower bound, and (iii) the series in (1.11) converges absolutely and uniformly for $-1 \leqq \sigma \leqq 2$. Other examples can be seen in a table of Mellin transforms [2].

The theory has a trivial extension to kernels of the form $(m / n)^{y} k^{\prime}(m, n)$ where $k^{\prime}$ satisfies our hypotheses, as is seen by applying to (1.1) the transformation $x_{n}=n^{\gamma} \xi_{n}$. Thus an infinity of the kernel at $n=0$ can be tolerated provided that it has a compensating zero at $m=0$; and vice versa.

1.3. Heuristic considerations. Dirichlet spaces. If we substitute from (1.4) into (1.1) it becomes formally

where

$$
x_{m}+\frac{1}{2 \pi i} \int_{0-i \infty}^{c+i \infty} K(s) m^{s-1} f_{N}(s) d s=g_{m}, \quad 0<c<1 .
$$

and thence, multiplying by $m^{-u}$ and adding,

$$
f_{N}(u)+\frac{1}{2 \pi i} \int_{c-i \infty}^{c+i \infty} K(s) f_{N}(s) \sum_{m=N}^{\infty} m^{s-1-u} d s=\sum g_{m} m^{-u} .
$$


These formalities will later be justified, but we may note immediately that the condition $\operatorname{Re} u>c$ is required so that the series in the integrand of (1.14) may converge.

It is familiar that, to obtain a workable theory of solution of a system such as (1.1), an a priori restriction on the sequences $\left\{x_{n}\right\}$ to be considered is necessary: we search for solutions in an assigned sequence-space. The preceding formulae show that, for our problem, the relevant spaces are what we call Dirichlet spaces $D_{\alpha}$, with the definition

$\left\{x_{n}\right\}$ lies in $D_{a}$ if the Dirichlet series $\sum x_{n} n^{-8}$ converges whenever $\sigma>\operatorname{Re} \alpha$.

Thus $D_{\alpha}=D_{\beta}$ if $\alpha, \beta$ have the same real part, and $D_{\alpha} \subset D_{\beta}$ if $\operatorname{Re} \alpha<\operatorname{Re} \beta$.

The Dirichlet spaces present a notable contrast to Hilbert space and its usual generalizations, in that their definition involves $\left\{x_{n}\right\}$ rather than $\left\{\left|x_{n}\right|\right\}$. We use them only descriptively, and do not need to norm them.

The values of $\alpha$ that are likely to be relevant may be seen by making a further formal step from (1.14). Here

$$
\sum_{N}^{\infty} m^{s-1-u}=\zeta(1+u-s)-\sum_{1}^{N-1} m^{s-1-u}=\zeta_{N}(1+u-s), \text { say, }
$$

where $\zeta(z)$ is Riemann's zeta function, which is everywhere continuable apart from the pole at $z=1$ where the residue is 1 . Thus (1.14) can be used to find the analytic continuation of $f_{N}(u)$ to any point to which $\sum g_{m} m^{-u}$ is continuable. Taking $c<\operatorname{Re} u<1$ we can displace the path across $s=u$ to a position $\sigma=c^{\prime}<1$, and for $\operatorname{Re} u<c^{\prime}(1.14)$ is equivalent to

$$
\{1+K(u)\} f_{N}(u)+\frac{1}{2 \pi i} \int_{0^{\prime}-i \infty}^{c^{\prime}+i \infty} K(s) f_{N}(s) \zeta_{N}(1+u-s) d s=\sum g_{m} m^{-u}
$$

whence we must expect $f_{N}(u)$ to have poles at all zeros of $1+K(u)$ which are to the left of $\sigma=c$. Since at such a pole of $f_{N}(u)$ the series (1.13) which originally defined it ${ }^{2}$ cannot converge, we see that the relevant values of $\alpha$ are the zeros of $1+K(u)$ whose real parts are between 0 and 1 , or $\alpha=0$ if there are no such zeros. The logical sequence is that we begin by choosing such an $\alpha$, and confine the search for solutions $\left\{x_{n}\right\}$ to those that are in $D_{\alpha}$. Then (1.13) defines $f_{N}(s)$ for $\sigma>\operatorname{Re} \alpha$, and - as will be proved in $\S 3.2-$ (1.1) implies (1.12), (1.14) with $c$ chosen between $\operatorname{Re} \alpha$ and 1 ; the restriction $c<1$ arises from (1.4). The passage from (1.1) to (1.14) may be reversed by taking (1.12) as a definition of $x_{m}$, and if - as will be proved - (1.14) has solutions $f_{N}(s)$ these yield solutions $\left\{x_{m}\right\}$ in $D_{\alpha}$. below.

2 The dummy symbol $u$ is equivalent to $s$. Similar switches in dummies will often occur 
There is here a slight air of paradox. If $\operatorname{Re} \alpha^{\prime}<\operatorname{Re} \alpha$, any solution in $D_{\alpha^{\prime}}$ is in $D_{\alpha}$ and hence is obtainable via (1.14) with $c>\operatorname{Re} \alpha$; but the corresponding $f_{N}(u)$ is regular at $\alpha$, in spite of the suggestion from (1.16) that it is there singular. The answer is that the suggestion must be wrong. In fact, in the case that is contemplated (1.14) has a solution depending linearly on at least one arbitrary constant, and a particular choice of this constant renders $f_{N}(u)$ regular at $\alpha$ and gives a solution $\left\{x_{n}\right\}$ which is in the more restricted space $D_{\alpha^{\prime}}$.

1.4. Treatment of the integral equation (1.14). Since the principal part of $\sum m^{s-1-u}$ for $u$ near $s$ is $1 /(u-s)$, we would obtain a singular integral equation with Cauchy-type kernel by making $u$ move on to the path of integration; but this equation is not quite of the standard type, on account of the residual singularity at $s=\infty$. Also the emphasis for us is on analytic properties of $f_{N}(s)$ rather than its properties on an assigned path of integration. Hence we give a treatment from first principles, in which $u$ is always excluded from the paths of integration; we make no appeal to the results regarding singular integral equations, but we use the leading ideas of that theory [3].

The first of these ideas is to use a factorization

$$
1+K(s)=K_{+}(s) K_{-}(s)
$$

in which the zeros and singularities of $K_{+}(s)\left[K_{-}(s)\right]$ are just those of $1+K(s)$ to the left [right] of the line of integration $\sigma=c$ in (1.14), so that $K_{+}(s)\left[K_{-}(s)\right]$ is regular and non-zero for $\sigma>c[\sigma<c]$. Then (1.14) and (1.16) show that $K_{+}(u) f_{N}(u)$ is an analytic function with no singularities except those of $\sum g_{m} m^{-u}$, and this can be taken as motivation for the factorization. Of course, the factorization would also be the primary step in an attack by Wiener-Hopf technique, but the characteristic step in this technique - the determination of a function by appeal to Liouville's theorem - does not seem to be applicable to our problem.

The second idea is to derive from (1.14) an equation which is free from the potential singularity at $u=s$, by application to it of a suitably chosen integral operator. One way to do this is to form the first iterate of the equation, which has the same type of potential singularity as the original equation, so that by suitable combination of the iterate with the original the singularity can be eliminated. However, the critical point is that the passage from the original to the derived equation should be reversible, and for the procedure just suggested this is not the case. The fruitful operator to use (§4) is, effectively, derived from (1.14) by taking the singular part of its adjoint. 


\section{The characteristic functions}

For a given kernel $k(m, n)$ the characteristic functions are $K(s)$ as introduced in (1.5) and defined by (1.8), and $1+K(s)$.

2.1. Special cases. When the kernel is rational the formula (1.9) shows that $K(s)$ has poles at most at $s=0, \pm 1, \cdots$, but in special cases some of these points may not be poles. The most important of these cases are:

(i) If $k(m, n)$ has a $j$-fold zero at $m=0, K(s)$ is regular at $s=1,2, \cdots j$.

(ii) If $k(m, n)$ has an l-fold zero at $n=0, K(s)$ is regular at $s=0$, $-1, \cdots-l+1$.

These results follow from (1.8), where in case (i) $k(1, z)=O\left(z^{-j-1}\right)$ for $z \sim \infty$, and in case (ii) $k(1, z)$ has an $l$-fold zero at $z=0$.

In these cases, then, the band of regularity of $K(s)$ is $-l<\sigma<j+1$, and in (1.4) and consequential formulae the restriction $-l<c<j+1$ is sufficient.

2.2. Symmetry and pseudo-symmetry. We call $k$ pseudo-symmetric if

$$
k(m, n)=m^{j} n^{2} k^{\prime}(m, n) \quad \text { with } \quad k^{\prime}(m, n)=k^{\prime}(n, m)
$$

where $j, l$ are non-negative integers; if $j=l, k$ is symmetric. From the homogeneity with degree -1 of $k$ we have $k\left(1, z^{-1}\right)=z k(z, 1)$, while from $(2.1)$

$$
k(z, 1)=z^{j} k^{\prime}(z, 1)=z^{j} k^{\prime}(1, z)=z^{j-l} k(1, z) .
$$

Hence (1.8) gives, by a change of variable $z=u^{-1}$,

$$
\begin{aligned}
K(s) & =\int_{0}^{\infty} k\left(1, z^{-1}\right) z^{l-1-1} z^{s-1} d z \\
& =\int_{0}^{\infty} k(1, u) u^{j-l-s} d u=K(1+j-l-s) .
\end{aligned}
$$

This shows that $K(s)$ has a centre of symmetry at $s=\frac{1}{2}(1+i-l)$, a point which bisects the axis of the band of regularity found in $\S 2.1$.

2.3. The zeros and indices. The zeros of the characteristic function $1+K(s)$ will play an important part in the theory; they may be investigated by standard methods, into which we do not here enter. For a general theory we must allow that some of them may lie in the band of regularity $(0,1)$ [or $(-l, j+1)$ in the special case of $\S 2.1$ ]; on account of $(1.7)$ the number in any interior part of this band must be finite, and if $K(s)$ is meromorphic the same will hold for the complete band.

By lines $\sigma=$ const. through these zeros the band of regularity is divided into a number of strips $S$.

For any such $S$, its index $\mu$ relative to $1+K(s)$ is defined as the increment in $(2 \pi)^{-1} \arg (1+K(s))$ as $s$ runs from $i-\infty$ along any path in $S$ to $i \infty$; on account of (1.7) it is an integer (positive, negative or zero). 
For any two adjacent strips, the one on the right has the greater index, the difference being equal to the number of zeros of $1+K(s)$ on the common boundary, counted according to their multiplicity.

When the kernel is symmetric or pseudo-symmetric, and the centreline $\sigma=\frac{1}{2}(1+j-l)$ of the band of regularity of $1+K(s)$ contains no zeros of this function, (2.2) shows that the strip $S$ which contains this line has zero index; but if there are zeros on the line - necessarily even in number the strips on either side of it have non-zero indices $\pm \mu$, and there is no strip with zero index.

For the signs of the set of indices of all the strips there are 7 possible cases:

(i) all are +; (ii) all -; (iii) there is only one strip, with index 0 ; (iv) some are + and one 0 ; (v) some - and one 0 ; (vi) some + and some - but none 0 ; (vii) all of $+, 0,-$ occur.

The example $k(m, n)=A /(m+n / a)$, where by $(1.11)$

$$
1+K(s)=1+\pi A a^{s} \operatorname{cosec} \pi s
$$

gives each of the cases for suitably chosen real $A$, $a$, with $a>0$ :

For $a=1$ we have case (iii) for $0<\pi A<1$, (vii) for $-1<\pi A<0$, and (vi) for $\pi A \leqq-1$. For $a \neq 1$ we take $A>0$ and note that, on the imaginary axis $s=i t$,

$$
1+K(i t)=1+\pi A \operatorname{cosech} \pi t \exp \left(i t \log a-\frac{1}{2} i \pi\right) .
$$

The two points where $\sinh \pi t= \pm \pi A$ divide the axis into three parts. On each of the extreme parts $\mid \pi A$ cosech $\pi t \mid<1$, and

$$
|\Delta \arg (1+K(i t))| \leqq \frac{1}{2} \pi
$$

while on the middle part the difference between $\arg (1+K(i t))$ and $\arg \exp (i t \log a)$ is always between $\pm \pi$. Hence for the whole axis $\sigma=0$, indented in either sense around the pole $s=0$, the index differs by at most 2 from

$$
\pi^{-2} \log a \operatorname{arcsinh}(\pi A) .
$$

For the line $\sigma=1$, indented around the pole $s=1$, the corresponding estimate is

$$
\pi^{-2} \log a \operatorname{arcsinh}(\pi a A) .
$$

For fixed positive $A$, both (2.3) and (2.4) are large positive when $a$ is sufficiently large, and the latter is much the larger; so there are a large number of strips all of positive index. Similarly, for fixed $A a$ they are both large negative when $a$ is sufficiently near 0 , and there are a large number 
of strips all of negative index. Thus we get cases (i) and (ii). Case (iv) arises from case (i) by keeping $A$ fixed and allowing $a$ to diminish continuously until the leftmost strip acquires the index 0 ; and case (v) similarly arises from case (ii).

2.4. Factorization. A factorization of $1+K(s)$ such as is indicated in (1.17) is relative to a zero-free strip as just defined; the factorizations relative to two such strips are different, but simply related.

(i) If the index of the strip is 0 we choose two paths $\sigma=c, c^{\prime}$ in the strip, with $c<c^{\prime}$, and define

$$
\begin{array}{ll}
K_{+}(s)=\exp \left[\frac{1}{2 \pi i} \int_{c-i \infty}^{c+i \infty} \frac{\log (1+K(z)) d z}{s-z}\right] & \text { for } \sigma>c, \\
K_{-}(s)=\exp \left[-\frac{1}{2 \pi i} \int_{c^{\prime}-i \infty}^{c^{\prime}+i \infty} \frac{\log (1+K(z)) d z}{s-z}\right] & \text { for } \sigma<c^{\prime} .
\end{array}
$$

Here, with an appeal to (1.7), we take the logarithm to be that branch which $\sim K(z)=O\left(e^{-\lambda|t|}\right)$ for $t=\operatorname{Im} z \sim-\infty$; and because the index is 0 the logarithm will be $O\left(e^{-\lambda \mid t^{3}}\right)$ for $t \sim+\infty$ also. Hence the integrals converge, and define regular functions of $s$ in the respective half-planes; so $K_{+}(s), K_{-}(s)$ are there regular and never zero.

For $c<\sigma<c^{\prime}$ both $K_{+}(s), K_{-}(s)$ are defined, and their product is the exp of a 'closed contour' integral, with the pole $z=s$ of the integrand inside. Hence

$$
K_{+}(s) K_{-}(s)=1+K(s) .
$$

By appeal to analytic continuation, this relation now serves to define each of $K_{+}, K_{-}$in the half-plane where originally the other was defined, in so far as $K(s)$ can there be continued. The relation is then universally valid, and shows that $K_{+}(s)\left[K_{-}(s)\right]$ has just those zeros and singularities of $1+K(s)$ as lie to the left [right] of the strip.

In (2.5) we can move the path of integration a little to the left, and the formula shows that

$K_{+}(s)$ and its reciprocal are bounded for $\sigma \geqq c$ and $1+O\left(\sigma^{-1}\right)$ uniformly for $\sigma \sim+\infty$.

Similarly

$K_{-}(s)$ and its reciprocal are bounded for $\sigma \leqq c^{\prime}$ and $1+O\left(\sigma^{-1}\right)$ uniformly for $\sigma \sim-\infty$.

If (1.7) holds in sectors centred on arg $s= \pm \frac{1}{2} \pi$, and not simply in a strip, these asymptotic estimates may be strengthened to asymptotic expansions in powers of $s^{-1}$; in the case of (2.5) this is done by rotating the ends of the 
path of integration as as to lie along rays $\arg z= \pm\left(\frac{1}{2} \pi+\delta\right), \delta>0$, and expanding $(s-z)^{-1}$ in powers of $s^{-1}$.

(ii) If the index $\mu$ of the strip is not zero we choose any points $s_{1}, s_{2}$ to the right and left of the strip, respectively, and introduce the auxiliary function

$$
\psi(s)=(1+K(s))\left(s-s_{1}\right)^{\mu}\left(s-s_{2}\right)^{-\mu} .
$$

Since the index of $\left(s-s_{1}\right) /\left(s-s_{2}\right)$ for the strip is -1 , the index of $\psi(s)$ is 0 , so there is a factorization $\psi(s)=\psi_{+}(s) \psi_{-}(s)$ with the properties just demonstrated. Thence we define

$$
K_{+}(s)=\psi_{+}(s)\left(s-s_{2}\right)^{\mu}, \quad K_{-}(s)=\psi_{-}(s)\left(s-s_{1}\right)^{-\mu},
$$

and $K_{+}(s), K_{-}(s)$ have the same properties, including (2.7), as in (i), except that

$$
\left\{\begin{array}{l}
(s+1-c)^{-\mu} K_{+}(s) \text { and its reciprocal are bounded for } \sigma \geqq c \text { and } \\
1+O\left(\sigma^{-1}\right) \text { uniformly for } \sigma \sim+\infty, \\
\left(s-1-c^{\prime}\right)^{\mu} K_{-}(s) \text { and its reciprocal are bounded for } \sigma \leqq c^{\prime} \text { and } \\
1+O\left(\sigma^{-1}\right) \text { uniformly for } \sigma \sim-\infty .
\end{array}\right.
$$

By Liouville's theorem it can be proved that these factor-functions are independent of the choice of the auxiliary points $s_{1}, s_{2}$.

If $(2.7)$ is the factorization relative to one strip, and if $1+K(s)$ has zeros $s_{1}, \cdots s_{r}$ on its right hand boundary, then the factorization relative to the next strip to the right must be

$$
1+K(s)=K_{+}(s) \prod_{v=1}^{r}\left(s-s_{v}\right) \cdot K_{-}(s) \prod_{v=1}^{r}\left(s-s_{v}\right)^{-1}=\bar{K}_{+}(s) \cdot \bar{K}_{-}(s) ;
$$

for the factors $\bar{K}_{+}, \bar{K}_{-}$have the correct disposition of zeros and singularities, and the correct normalization as shown in (2.8).

\section{Convergence and commutation theorems}

To justify the formal passage in $\S \mathbf{1 . 3}$ from the linear system (1.1) to the integral equation (1.14) we have to establish the validity of certain commutations of summation and integration, involving Dirichlet series. For this we appeal as necessary to the well known properties [4]:

if $\sum x_{n} n^{-8}$ converges for $\sigma>\sigma_{0}$, then (i) it converges uniformly in any compact part of the half plane $\sigma \geqq \sigma_{0}+\varepsilon>\sigma_{0}$, and defines a function $f(s)$ which is regular and uniformly $O(t)$ for $|t| \sim \infty$ in this half plane; and (ii) the series converges absolutely and uniformly in the half plane $\sigma \geqq \sigma_{0}+1+\varepsilon$, and $f(s)$ is there bounded. 
3.1. LEMMA 1. Let $\psi(s)$ be regular in a strip $\alpha<\sigma<\beta$, and for $|t| \sim \infty$ in the strip $\alpha<\sigma<1+\beta$ let $\psi(s)$ be regular and $O\left(e^{-\lambda|t|}\right)$ where $\lambda>0$, and let $\left\{x_{n}\right\}$ be in the Dirichlet space $D_{\alpha}$; then for $\alpha<c<\beta$

$$
\sum x_{n} \int_{c-i \infty}^{c+i \infty} \psi(s) n^{-s} d s=\int_{c-i \infty}^{c+i \infty} \psi(s) \sum x_{n} n^{-s} d s,
$$

the series on the left being necessarily convergent.

Proof. Since $\left\{x_{n}\right\}$ is in $D_{a}, \sum x_{n} n^{-s}$ converges for $\sigma \geqq c$ and converges absolutely for $\sigma \geqq c+1$. Let the path $\sigma=c$ be deformed into a position $L$ in which the sufficiently distant parts lie on $\sigma=c+1$; by the hypothesis on $\psi(s)$ the deformation will not involve the crossing of any singularities of this function. Then for integration along the far parts of $L$ we have, by absolute convergence,

$$
\sum x_{n} \int \psi(s) n^{-s} d s=\int \psi(s) \sum x_{n} n^{-s} d s,
$$

and by uniform convergence the same holds for integration along the intervening part. Hence the analogue of (3.1), in which the integrations are along $L$, is valid.

But since $\left|n^{-s}\right| \leqq n^{-c}$ and $\left|\sum x_{n} n^{-s}\right| \leqq A|t|$ and $\psi(s)=O\left(e^{-\lambda|t|}\right)$, each of the integrals along $L$ is equal to the corresponding integral along $\sigma=c$; so (3.1) is established.

3.2. The integral equation equivalent to (1.1). The passage from (1.1) via (1.4) to (1.12) involves the commutation (3.1), with $\psi(s)=K(s) m^{2-1}$; so (1.12) is established provided $\left\{x_{n}\right\}$ is in some $D_{\alpha}$ with $\alpha<1$, and $\alpha<c<1 .^{3}$ To pass thence to (1.14) we multiply (1.12) by $m^{-u}$, sum, and commute summation with integration. This commutation is justified by absolute convergence, provided $\operatorname{Re} u>c$, so (1.14) is established, with the series $\sum g_{m} m^{-u}$ on the right necessarily convergent - which means that (1.1) cannot be satisfied by an $\left\{x_{n}\right\}$ in $D_{\alpha}$ unless $\left\{g_{n}\right\}$ is in $D_{\alpha}$.

Conversely, suppose that $\left\{g_{n}\right\}$ is in $D_{\alpha}$ and that (1.14) has a solution $f_{N}(u)$ which is analytic for $\operatorname{Re} u>c$, and continuous up to $\operatorname{Re} u=c$, and $O(\operatorname{Im} u)^{A}$ for some constant $A$ and $\operatorname{Re} u \geqq \alpha+\varepsilon>\alpha$. Then $f_{N}(u)$ satisfies also (1.16); and if $1+K(u) \neq 0$ for $\alpha<\operatorname{Re} u<c^{\prime}, f_{N}(u)$ is regular for $\operatorname{Re} u>\alpha$.

Let $x_{m}$ be defined by (1.12), viz.

$$
x_{m}+\frac{1}{2 \pi i} \int_{c-i \infty}^{c+i \infty} K(s) m^{s-1} f_{N}(s) d s=g_{m}
$$

for $m=N, N+1, \cdots$. By the hypotheses on $f_{N}(s)$ we can here move the line of integration to any position $\sigma=\sigma_{1}>\alpha$, and for such a $\sigma_{1}$ the integral

${ }^{3} \alpha<c<j+1$ in the special case of $\S 2.1$. 
is $O\left(m^{\sigma_{1}-1}\right)$; also $\sum g_{m} m^{-\sigma_{1}}$ converges since $\left\{g_{m}\right\}$ is in $D_{\alpha}$. Hence $\sum x_{m} m^{-s}$ converges for $\sigma>\sigma_{1}$, where $\sigma_{1}-\alpha$ may be arbitrarily small; so $\left\{x_{n}\right\}$ is in $D_{\alpha}$. By multiplying (3.2) by $m^{-u}$, summing over $m$, and subtracting (1.14) we get

$$
\sum_{N}^{\infty} x_{m} m^{-u}=f_{N}(u)
$$

for $\operatorname{Re} u>c$, and by continuation this holds for $\operatorname{Re} u>\alpha$. Finally, by substituting from (3.3) into (3.2) and commuting the summation and integration we arrive via (1.4) back at (1.1). To sum up:

THEOREM 1. For some $\alpha$ in the band of regularity, say $(0,1)$ of $K(s)$ let $\left\{g_{n}\right\}$ be in $D_{\alpha}$, and let $1+K(s) \neq 0$ in the strip $\alpha<\sigma \leqq c<1$. Then the formulae (3.2), (3.3) establish a one-one correspondence between solutions $\left\{x_{n}\right\}$ of (1.1) that are in $D_{\alpha}$ and solutions $f_{N}(u)$ of

$$
f_{N}(u)+\frac{1}{2 \pi i} \int_{c-i \infty}^{c+i \infty} K(s) \zeta_{N}(1+u-s) f_{N}(s) d s=\sum_{N}^{\infty} g_{n} n^{-u}=g_{N}(u)
$$

$\operatorname{Re} u>c$

which are regular for $\operatorname{Re} u>\alpha$ and $O(\operatorname{Im} u)^{A}$ for $\operatorname{Re} u \geqq \alpha+\varepsilon>\alpha$. Here

$$
\zeta_{N}(z)=\zeta(z)-\sum_{1}^{N-1} n^{-z}
$$

3.3. The adjoint system and integral equation. If we compound the equations (1.1) with a set of multipliers $y_{m}$ we obtain

$$
\sum h_{n} x_{n}=\sum y_{m} g_{m}
$$

where

provided that

$$
y_{n}+\sum_{m=N}^{\infty} y_{m} k(m, n)=h_{n}
$$

$$
\sum y_{m} x_{m} \text { converges }
$$

and

$$
\sum_{m} y_{m} \sum_{n} k(m, n) x_{n}=\sum_{n} x_{n} \sum_{m} y_{m} k(m, n) \text {. }
$$

The utility of this procedure is that (3.6) may be a 'nicer' equation than any one of (1.1); e.g. the left member reduces to $x_{\nu}$ if $h_{n}=\delta_{n \nu}$ in the adjoint system (3.7). Taking $h_{n}=0,(3.6)$ leads to the theorem that for (1.1) to have any solution $\left\{x_{n}\right\}$ it is necessary that $\left\{g_{m}\right\}$ be orthogonal to every solution $\left(y_{m}\right)$ of the homogeneous adjoint system ${ }^{4}$ - provided of course that the

4 The notation ( ) is used for row-vectors, \{ \} for column-vectors. 
conditions (3.8), (3.9) are satisfied. In fact, the orthogonal condition is sufficient also for solubility of $(1.1)$, but the proof of this requires much more effort, and is achieved only in $\S 6$.

To perform on (3.7) operations that are strictly analogous to those of $\S 1.3$ we should begin by making in (1.4) the change of variable $s^{\prime}=1-s$, so that the index of the summation variable $m$ may become $-s^{\prime}$. This shows that the adjoint characteristic function is $K(1-s)$, and that properties relative to left half-planes are adjoint to those for right half-planes. However, when simultaneous consideration of the direct and adjoint systems is in question it is clearest to apply (1.4) as it stands to both. Hence we are led to the following adjoint of Theorem 1;

THEOREM IA. Let $\alpha<\sigma<\beta$ be a zero-free strip of regularity of $1+K(s)$, and let $\left(h_{n}\right)$ be in $D_{1-\beta}$. Then if $\alpha<c^{\prime}<\beta$ the formulae

$$
f_{N}^{*}(u)=\sum y_{m} m^{u-1}, \quad y_{n}+\frac{1}{2 \pi i} \int_{c^{\prime}-i \infty}^{c^{\prime}+i \infty} K(s) n^{-s} f_{N}^{*}(s) d s=h_{n}
$$

establish a one-one correspondence between solutions $\left(y_{n}\right)$ of $(3.7)$ that are in $D_{1-\beta}$ and solutions $f_{N}^{*}(u)$ of

$$
f_{N}^{*}(u)+\frac{1}{2 \pi i} \int_{c^{\prime}-i \infty}^{c^{\prime}+i \infty} K(s) \zeta_{N}(1-u+s) f_{N}^{*}(s) d s=\sum h_{n} n^{u-1}=h_{N}(u),
$$

$\operatorname{Re} u<c^{\prime}$

which are regular for $\operatorname{Re} u<\beta$ and $O(\operatorname{Im} u)^{A}$ for $\operatorname{Re} u \leqq \beta-\varepsilon<\beta$.

3.4. Further convergence theorems. We now consider propositions of the form (3.8) and (3.9). The example $x_{m}=y_{m}=(-1)^{m}$ shows that the presence of $\left\{x_{m}\right\},\left(y_{m}\right)$ in $D_{0}-$ and a fortiori in $D_{\alpha}$ with $\alpha>0-$ is insufficient to secure the convergence of $\sum x_{m} y_{m}$. However, the $x_{m}, y_{m}$ that will be in question will satisfy (1.1) or (3.7) or some set of equations such as (3.6) that are found a posteriori, and this is a decisive restriction.

LEMмA 2. Let $\alpha, \beta$ be respectively to the left and right of a zero-free strip of regularity of $1+K(s)$, let $\left\{x_{n}\right\}$ be in $D_{\alpha}$, and let $\left(y_{n}\right)$ be a solution in $D_{1-\beta}$ of (3.7). Then $\sum x_{n} y_{n}$ converges if $\sum h_{n} x_{n}$ converges and if either (i) $\sum x_{n} n^{-s}$ converges absolutely for $\sigma>\alpha$, or (ii) $\sum h_{n} n^{s-1}$ has a regular continuation into the parts of the strip $\beta \leqq \sigma<\beta+1$ for which $|t|$ is sufficiently large and is there at worst $O|t|^{A}$ for $|t| \sim \infty$, uniformly in $\sigma$.

Proof. The hypothesis on $y_{n}$ implies (3.10), so from the convergence of $\sum x_{n} h_{n}$ will follow that of $\sum x_{n} y_{n}$ provided

$$
\sum x_{n} \int_{c^{\prime}-i \infty}^{c^{\prime}+i \infty} K(s) n^{-s} f_{N}^{*}(s) d s
$$

converges. If $\sum x_{n} n^{-8}$ converges absolutely this is immediate, since 
$K(s) f_{N}^{*}(s)$ is exponentially small for $t \sim \pm \infty$. If the convergence is only conditional we appeal to Lemma 1 , for which we require the continuability of $f_{N}^{*}(s)$ through $c^{\prime} \leqq \sigma \leqq c^{\prime}+1$ with $|t|$ sufficiently large; and for this latter we have from (3.11)

$$
(1+K(u)) f_{N}^{*}(u)+\frac{1}{2 \pi i} \int_{c-i \infty}^{c+i \infty} K(s) \zeta_{N}(1-u+s) f_{N}^{*}(s) d s=\sum h_{n} n^{u-1}
$$

for $\alpha<c<c^{\prime}$ and $\operatorname{Re} u>c$. The hypothesis (ii) on $h_{n}$, together with $K(u) \sim 0$ for $\operatorname{Im} u \sim \pm \infty$, shows thence that $f_{N}^{*}(u)$ is continuable as desired, the integral term being at worst algebraically large for $\operatorname{Im} u \sim \pm \infty$ (from known properties of $\zeta(z)$, cf. $\S 5.1$ ).

CoROllary. If $\left(y_{n}\right)$ is a solution in $D_{1-\beta}$ of the homogeneous adjoint equation, it is sufficient that $\left\{x_{n}\right\}$ be in $D_{\alpha}$ : Proof: $h_{n}=0$.

We now deal with the commutation property (3.9), supposing that $\left\{x_{n}\right\}$ is in $D_{\alpha}$ and $\left(y_{n}\right)$ in $D_{1-\beta}$, where $\alpha, \beta$ are as in Lemma 2 . On substitution for $k(m, n)$ from (1.4) the sum on the left becomes, by use of Lemma 1 ,

$$
\frac{1}{2 \pi i} \sum y_{m} \int_{c-i \infty}^{c+i \infty} K(s) m^{s-1} f_{N}(s) d s, \quad f_{N}(s)=\sum x_{n} n^{-8}
$$

and this converges and equals

$$
\frac{1}{2 \pi i} \int K(s) f_{N}^{*}(s) f_{N}(s) d s, \quad f_{N}^{*}(s)=\sum y_{m} m^{s-1}
$$

provided $\sum y_{m} m^{s-1}$ is a finite summation or is absolutely convergent; or provided the adjoint of the argument of Lemma 1 is applicable, which involves a displacement of the far parts of the path to the left by one unit, and so involves the leftward continuation of $f_{N}(s)$. For this latter the adjoint of the argument of Lemma 2 will serve, provided $\left\{x_{n}\right\}$ satisfies (1.1) with $\left\{g_{n}\right\}$ suitably restricted.

The commutative property (3.9) will now follow from the symmetry of the first expression in (3.12), under adjoint hypotheses to those just indicated. Hence we obtain

LeMMA 3. Let $\alpha, \beta$ be respectively to the left and right of a zero-free strip of regularity of $1+K(s)$, let $\left\{x_{n}\right\}$ be in $D_{\alpha}$ and $\left(y_{n}\right)$ in $D_{1-\beta}$ and let $\alpha<c<\beta$. Then (3.9) is valid provided

(i) $\sum y_{m} m^{c-1}$ is absolutely convergent, or $\left\{x_{n}\right\}$ satisfies (1.1) with $\sum g_{n} n^{-s}$ continuable into the parts of the strip $\alpha-1<\sigma<\alpha$ for which $|t|$ is sufficiently large and uniformly $O\left(\left.|t|\right|^{A}\right)$ for $|t| \sim \infty$ there; and

(ii) $\sum x_{m} m^{-c}$ is absolutely convergent, or $\left(y_{n}\right)$ satisfies (3.7) with $\sum h_{n} n^{8-1}$ continuable into $\beta \leqq \sigma<\beta+1$ and uniformly $O\left(|t|^{A}\right)$ for $|t| \sim \infty$ there. 
If the $y$-summation is finite the hypothesis (ii) is unnecessary and (i) is satisfied.

Note. In the case of $h_{n}=k(m, n)$ with $m$ fixed - which will arise in $\S 6$ - we can by use of (1.4) express $\sum h_{n} n^{s-1}$ as an integral and hence show that the continuation hypothesis in (ii) is satisfied; while for $h_{n}=0$ this is trivial. Similarly (i) is satisfied if $g_{m}=0$ or $g_{m}=k(m, n)$ with $n$ fixed.

\section{Reduction of the integral equation (3.4)}

The heuristic considerations underlying the following investigation have been shown in $\S 1.4$.

4.1. The kernel of (3.4) contains the singular factor $\zeta_{N}(1+u-s)$, and to isolate its singular part we put

$$
\zeta_{N}(z)=\left(N-\frac{1}{2}\right)^{1-z}\left(\frac{1}{z-1}+\eta_{N}(z)\right)
$$

so that $\eta_{N}(z)$ is everywhere regular. The dependence of this function on $N$ will be discussed in $\S 5$; at present all that we need is that it is $O_{N}(\operatorname{Im} z)$ on paths of integration where $\operatorname{Re} z$ is near 1 , so that integrals where it is multiplied by an exponentially small factor converge absolutely.

In (3.4) we take the path $\sigma=c$ to lie in a zero-free strip of $1+K(s)$, of index $\mu$, relative to which there is the factorization

$$
1+K(s)=K_{+}(s) K_{-}(s)
$$

with the properties shown in $\S 2.4$.

To match (4.1) we put

$$
K_{+}(s) f_{N}(s)=\left(N-\frac{1}{2}\right)^{-s} f(s), \quad g_{N}(s)=\left(N-\frac{1}{2}\right)^{-s} g(s) ;
$$

the effect of the factors $\left(N-\frac{1}{2}\right)^{-8}$ here and in (4.1) is to absorb the grossest part of the variation of $f_{N}(s)$. By these transformations (3.4) becomes

$$
\frac{f(u)}{K_{+}(u)}+\frac{1}{2 \pi i} \int_{L}\left(K_{-}(s)-\frac{1}{K_{+}(s)}\right)\left(\frac{1}{u-s}+\eta_{N}(1+u-s)\right) f(s) d s=g(u)
$$

where $L$ is the path $\sigma=c$, for $u$ to the right of $L$.

4.2. For logical clarity we define, for $s$ to the right of $L$,

$$
F(s)=\frac{f(s)}{K_{+}(s)}+\frac{1}{2 \pi i} \int_{L}\left(K_{-}(z)-\frac{1}{K_{+}(z)}\right)\left(\frac{1}{s-z}+\eta_{N}(1+s-z)\right) f(z) d z-g(s),
$$

a functional of any two functions $f(s), g(s)$ which are regular in the strip 
containing $L$ and are uniformly there $O|t|^{A}$ for $|t| \sim \infty, A$ being some constant. Let $L^{\prime}$ be a path $\sigma=$ const. in the strip, to the right of $L$; then by (4.5) and (2.8) $F(s)$ is defined and regular on $L^{\prime}$, and is there $O\left(t^{A+\mid \mu^{\prime}}\right)$; so for $u$ not on $L^{\prime}$

$$
\begin{aligned}
\int_{L^{\prime}}( & \left.K_{+}(s)-\frac{1}{K_{-}(s)}\right) \frac{F(s) d s}{s-u} \\
& =\int_{L^{\prime}}\left(1-\frac{1}{K_{+}(s) K_{-}(s)}\right) \frac{f(s) d s}{s-u}-\int_{L^{\prime}}\left(K_{+}(s)-\frac{1}{K_{-}(s)}\right) \frac{g(s) d s}{s-u} \\
& +\int_{L}\left(K_{-}(z)-\frac{1}{K_{+}(z)}\right) \frac{f(z) d z}{2 \pi i} \int_{L^{\prime}}\left(K_{+}(s)-\frac{1}{K_{-}(s)}\right) \eta_{N}(1+s-z) d s \\
& +\int_{L}\left(K_{-}(z)-\frac{1}{K_{+}(z)}\right) f(z) d z \int_{L^{\prime}}\left(K_{+}(s)-\frac{1}{K_{-}(s)}\right) \frac{d s}{2 \pi i(s-u)(s-z)}
\end{aligned}
$$

by (1.7), (2.7) and (2.8) all the integrands here are exponentially small at infinity, so the commutations that have been imposed on the double integrals are justified by absolute convergence.

We wish now to handle the inner integral in the last term by splitting it into two integrals, involving $K_{+}(s), 1 / K_{-}(s)$ separately, and evaluating these from residues in the half planes to the right and left of $L^{\prime}$ where these respective functions are regular. From (2.8) this can be done provided the index $\mu$ is zero or negative, and when $u$, like $z$, is to the left of $L^{\prime}$ the resulting value of the last term in (4.6) is

$$
-\int_{L}\left(K_{-}(z)-\frac{1}{K_{+}(z)}\right) \frac{f(z) d z}{K_{-}(z)(z-u)}-\int_{L}\left(K_{-}(z)-\frac{1}{K_{+}(z)}\right) \frac{f(z) d z}{K_{-}(u)(u-z)} .
$$

The first of these terms agrees with the first term on the right of (4.6) except that the respective paths of integration are $L, L^{\prime}$; so taking now $u$ to be between these paths they combine to give, by the residue at $s=u$,

$$
2 \pi i\left(1-\frac{1}{K_{+}(u) K_{-}(u)}\right) f(u)
$$

The second of the terms, when multiplied by $(2 \pi i)^{-1} K_{-}(u)$, coincides with a term in the expression (4.5) for $F(u)$ and can thence be eliminated. We state the result with the help of an auxiliary function $G(u)$, as follows:

if $f(s), g(s)$ are regular in the strip containing $L, L^{\prime}$ and are uniformly $O\left(t^{A}\right)$ for $t \sim \pm \infty$, and if $u$ is between $L, L^{\prime}$ and the index $\mu$ of the strip is not positive, then the statements 


$$
\begin{aligned}
G(u)= & \frac{F(u)}{K_{-}(u)}+\frac{1}{2 \pi i} \int_{L^{\prime}}\left(K_{+}(s)-\frac{1}{K_{-}(s)}\right) \frac{F(s) d s}{s-u}, \\
G(u)= & f(u)-\frac{g(u)}{K_{-}(u)}-\frac{1}{2 \pi i} \int_{L^{\prime}}\left(K_{+}(s)-\frac{1}{K_{-}(s)}\right) \frac{g(s) d s}{s-u} \\
& +\frac{1}{2 \pi i} \int_{L}\left(K_{-}(z)-\frac{1}{K_{+}(z)}\right) f(z) d z\left\{\frac{\eta_{N}(1+u-z)}{K_{-}(u)}\right. \\
& \left.+\int_{L^{\prime}}\left(K_{+}(s)-\frac{1}{K_{-}(s)}\right) \frac{\eta_{N}(1+s-z) d s}{2 \pi i(s-u)}\right\}
\end{aligned}
$$

are equivalent, where $F(s)$ is defined by (4.5).

4.3. When $\mu>0$ we take $\mu+1$ distinct points $u_{0}, u_{1}, \cdots u_{\mu}$ between $L$ and $L^{\prime}$, put $u=u_{r}$ in (4.6) and multiply it by the reciprocal of $\prod_{p \neq r}^{\prime}\left(u_{r}-u_{p}\right)$, and add the results. Since

$$
\frac{1}{\prod_{r=0}^{\mu}\left(s-u_{r}\right)}=\sum_{r=0}^{\mu} \frac{1}{\left(s-u_{r}\right) \prod_{p \neq r}^{\prime}\left(u_{r}-u_{p}\right)},
$$

reference to (2.8) shows that we shall obtain in the last term an inner integrand which is $O\left(s^{-2}\right)$, so it can be evaluated as before, and the result can be expressed in partial fraction form as on the right of (4.9). This form is maintained in the subsequent eliminations of integrals via the $F\left(u_{r}\right)$, and the result is, with $G(u)$ defined by $(4.8)$,

$$
\begin{aligned}
& \sum_{r=0}^{\mu} \frac{G\left(u_{r}\right)}{\prod_{p \neq r}^{\prime}\left(u_{r}-u_{p}\right)} \\
& \quad=\sum_{r=0}^{\mu} \frac{1}{\prod_{p \neq r}^{\prime}\left(u_{r}-u_{p}\right)}\left\{\frac{F\left(u_{r}\right)}{K_{-}\left(u_{r}\right)}+\frac{1}{2 \pi i} \int_{L^{\prime}}\left(K_{+}(s)-\frac{1}{K_{-}(s)}\right) \frac{F(s) d s}{s-u_{r}}\right\} .
\end{aligned}
$$

4.4. Returning to the case where $\mu=-\mu^{\prime}$ is negative, we could have used in (4.6) an arbitrary polynomial factor $P_{\mu^{\prime}-1}(s)$ of degree $\mu^{\prime}-1$ in place of the factor $(s-u)^{-1}$. Hence we obtain

$$
\begin{aligned}
\int_{L^{\prime}}\left(K_{+}(s)\right. & \left.-\frac{1}{K_{-}(s)}\right) P_{\mu^{\prime}-1}(s) F(s) d s \\
= & -\int_{L^{\prime}}\left(K_{+}(s)-\frac{1}{K_{-}(s)}\right) P_{\mu^{\prime}-1}(s) d s \\
& \cdot\left\{g(s)-\frac{1}{2 \pi i} \int_{L}\left(K_{-}(z)-\frac{1}{K_{+}(z)}\right) \eta_{N}(1+s-z) f(z) d z\right\}
\end{aligned}
$$


4.5. The operator on the right of (4.7) has the same structure relative to $L^{\prime}, K_{+}, K_{\text {- }}$ as that on the right of (4.5) has to $L, K_{-}, K_{+}$. Hence we can establish similar transformations, as follows:

if $F(s)$ is regular in the strip containing $L, L^{\prime}$ and uniformly $O\left(t^{A}\right)$ for $t \sim \pm \infty$, and $G(u)$ is thence defined by (4.7) for $u$ between $L, L^{\prime}$, then for $\mu \geqq 0$

$$
F(u)=\frac{G(u)}{K_{+}(u)}-\frac{1}{2 \pi i} \int_{L}\left(K_{-}(s)-\frac{1}{K_{+}(s)}\right) \frac{G(s) d s}{s-u},
$$

and if $\mu=-\mu^{\prime}<0$ and $u_{0}, u_{1}, \cdots u_{\mu^{\prime}}$ are in the strip,

$$
\begin{aligned}
& \sum_{r=0}^{\mu^{\prime}} \frac{F\left(u_{r}\right)}{\prod_{p \neq r}^{\prime}\left(u_{r}-u_{p}\right)}=\sum_{r=0}^{\mu^{\prime}} \frac{1}{\prod_{p \neq r}^{\prime}\left(u_{r}-u_{p}\right)} \\
& \cdot\left\{\frac{G\left(u_{r}\right)}{K_{+}\left(u_{r}\right)}-\frac{1}{2 \pi i} \int_{L}\left(K_{-}(s)-\frac{1}{K_{+}(s)}\right) \frac{G(s) d s}{s-u_{r}}\right\} .
\end{aligned}
$$

4.6. The integral equation (4.4) is by (4.5) equivalent to $F(s) \equiv 0$. By (4.7) this implies $G(u) \equiv 0$ when $\mu \leqq 0$, while for $\mu>0$ it implies by $(4.10)$

$$
\sum_{r=0}^{\mu} \frac{G\left(u_{r}\right)}{\prod_{p \neq r}^{\prime}\left(u_{r}-u_{p}\right)} \equiv 0,
$$

which shows that $G(u)$ is a polynomial $P_{\mu-1}(u)$ of degree $\mu-1$. Hence, referring to (4.8) and (4.11):

for $g(u)$ regular in the strip and $O(\operatorname{Im} u)^{A}$ for $u \sim \pm i \infty$, any solution $f(u)$ of (4.4) which is similarly behaved to $g(u)$ must satisfy, for $u$ between $L$ and $L^{\prime}$,

$$
\begin{aligned}
f(u)+ & \frac{1}{2 \pi i} \int_{L}\left(K_{-}(z)-\frac{1}{K_{+}(z)}\right) f(z) d z \\
& \cdot\left\{\frac{\eta_{N}(1+u-z)}{K_{-}(u)}+\int_{L^{\prime}}\left(K_{+}(s)-\frac{1}{K_{-}(s)}\right) \frac{\eta_{N}(1+s-z) d s}{2 \pi i(s-u)}\right\} \\
& =\frac{g(u)}{K_{-}(u)}+\frac{1}{2 \pi i} \int_{L^{\prime}}\left(K_{+}(s)-\frac{1}{K_{-}(s)}\right) \frac{g(s) d s}{s-u}+ \begin{cases}0 & \text { if } \mu \leqq 0 \\
P_{\mu-1}(u) & \text { if } \mu>0\end{cases}
\end{aligned}
$$

where $\mu$ is the index of the strip and $P_{\mu-1}$ is some polynomial of degree $\mu-1$; also if $\mu=-\mu^{\prime}<0, f(u)$ must satisfy

$$
\begin{aligned}
& \int_{L^{\prime}}\left(K_{+}(s)-\frac{1}{K_{-}(s)}\right) P_{\mu^{\prime}-1}(s) d s \\
& \cdot\left\{g(s)-\int_{L}\left(K_{-}(z)-\frac{1}{K_{+}(z)}\right) \frac{\eta_{N}(1+s-z) f(z) d z}{2 \pi i}\right\}=0
\end{aligned}
$$

for an arbitrary polynomial $P_{\mu^{\prime}-1}$ of degree $\mu^{\prime}-1$. 
In (4.14) we can allow $u$ to move on to the $z$-path $L$ since $\eta_{N}(1+u-z)$ is there regular and in the inner integral $|s-u|$ is bounded away from 0; and on account of the exponential smallness of $K_{-}(z)-1 / K_{+}(z)$ for $z \sim \pm i \infty$ this is a regular (Fredholm) equation. In $\S \mathbf{5}$ we shall prove that it is soluble by iteration when (for a given $k(m, n)$ ) $N$ is sufficiently large.

4.7. Conversely, we shall now prove that the solution of $(4.14)$ satisfies (4.4). We assume that $g(u), f(u)$ are regular in the strip and $O(\operatorname{Im} u)^{A}$ for $u \sim \pm i \infty$, the former by hypothesis and the latter consequentially, as will be shown in $\S 5$. Then by (4.5) $F(s)$ is defined and regular to the right of $L$; but for $s$ to the left of $L^{\prime}(4.5)$ implies

$F(s)=f(s) K_{-}(s)+\frac{1}{2 \pi i} \int_{L}\left(K_{-}(z)-\frac{1}{K_{+}(z)}\right)\left(\frac{1}{s-z}+\eta_{N}(1+s-z)\right) f(z) d z-g(s)$,

and from the two formulae it follows that $F(s)$ is regular in the whole strip and is there of the form $O\left(t^{A}\right)$ for $t \sim \pm \infty$. Hence we can appeal to (4.12) or $(4.13)$.

If $\mu=0,(4.14)$ is by (4.8) equivalent to $G(u) \equiv 0$, whence from (4.12) $F(u) \equiv 0$; so $f(u)$ satisfies (4.4).

If $\mu>0$ we can take $P_{\mu-1}$ in (4.14) to have arbitrary coefficients, and comparison with $(4.8)$ gives $G(u)=P_{\mu-1}(u)$. Thence by $(2.8)$

$$
K_{-}(s) G(s)=O\left(s^{-1}\right), \quad G(s) / K_{+}(s)=O\left(s^{-1}\right)
$$

in the appropriate half planes. Hence in (4.12) we can split the integral into two parts, and since $u$ is to the right of $L$ its value is $G(u) / K_{+}(u)$; so $F(u) \equiv 0$, as desired. It is plain that linear independence of a set of polynomials $P_{\mu-1}$ on the right of (4.14) implies linear independence of the corresponding solutions $f(u)$.

If $\mu<0$ we have $G(u) \equiv 0$, as when $\mu=0$, whence (4.13) shows that $F(u)$ is some polynomial of degree $\mu^{\prime}-1$. We assume that the consistency condition (4.15) is satisfied, and this along with (4.11) gives

$$
\int_{L^{\prime}}\left(K_{+}(s)-\frac{1}{K_{-}(s)}\right) \frac{s^{\mu^{\prime}} / u^{\mu^{\prime}}-1}{s-u} F(s) d s=0
$$

where we have put

$$
P_{\mu^{\prime}-1}(s)=\frac{s^{\mu^{\prime}} \mid u^{\mu^{\prime}}-1}{s-u}
$$

a polynomial involving $u$ as an arbitrary parameter. Thus (4.7), where $G(u) \equiv 0$, becomes 


$$
\begin{aligned}
-2 \pi i F(u) & =K_{-}(u) \int_{L^{\prime}}\left(K_{+}(s)-\frac{1}{K_{-}(s)}\right) \frac{F(s) d s}{s-u} \\
& =\frac{K_{-}(u)}{u^{\mu^{\prime}}} \int_{L^{\prime}}\left(K_{+}(s)-\frac{1}{K_{-}(s)}\right) \frac{s^{\mu^{\prime}} F(s) d s}{s-u} .
\end{aligned}
$$

This is valid for $\operatorname{Re} u \sim-\infty$. But then, on the right, $K_{-}(u)=O\left(u^{\mu^{\prime}}\right)$ and the integral is $O(\operatorname{Re} u)^{-1}$; so since $F(u)$ is a polynomial it must be 0 , as desired.

4.8. By theorem 1 ( $(3.2)$ each solution of (4.4) leads to a solution of (1.1). Before we give a formal statement of the results we shall verify that when $\mu<0$ the consistency condition (4.15) is (as we would expect) equivalent to the orthogonality of $\left\{g_{n}\right\}$ to every solution $\left(y_{n}\right)$ of the homogeneous adjoint system (3.7). On substituting in (3.11) for $\zeta_{N}$ from (4.1) and putting

$$
K_{-}(s) f_{N}^{*}(s)=\left(N-\frac{1}{2}\right)^{s} f^{*}(s)
$$

this equation, with $h_{n}=0$, becomes

$$
\frac{f^{*}(u)}{K_{-}(u)}+\frac{1}{2 \pi i} \int_{L^{\prime}}\left(K_{+}(s)-\frac{1}{K_{-}(s)}\right)\left(\frac{1}{s-u}+\eta_{N}(1-u+s)\right) f^{*}(s) d s=0
$$

and by arguments like those of $\S \S 4.2-4.7$ this is proved to be equivalent to

$$
\begin{aligned}
f^{*}(u) & +\int_{L^{\prime}}\left(K_{+}(z)-\frac{1}{K_{-}(z)}\right) \frac{f^{*}(z) d z}{2 \pi i} \\
& \cdot\left\{\frac{\eta_{N}(1-u+z)}{K_{+}(u)}-\int_{L}\left(K_{-}(s)-\frac{1}{K_{+}(s)}\right) \frac{\eta_{N}(1-s+z) d s}{2 \pi i(s-u)}\right\}=P_{\mu^{\prime}-1}(u)
\end{aligned}
$$

when $\mu=-\mu^{\prime}<0, P_{\mu^{\prime}-1}$ being an arbitrary polynomial of degree $\mu^{\prime}-1$. By the theory of $\S 5,(4.19)$ has a unique solution when $N$ is sufficiently large, and it is plain that the solutions corresponding to different polynomials $P_{\mu^{\prime}-1}$ are linearly independent. Hence, via (4.18) and $(3.10)$, we have $\mu^{\prime}$ solutions $\left(y_{n}\right)$ in $D_{1-\beta}$ of the homogeneous (3.7), linearly independent, for $\beta$ to the right of the strip in question.

For any such solution $\left(y_{n}\right)$ and any $\left\{g_{n}\right\}$ in $D_{\alpha}$, where $\alpha$ is to the left of the strip, the corollary to Lemma 2 along with (3.7) gives

$$
-\sum y_{n} g_{n}=\frac{1}{2 \pi i} \int_{L^{\prime}} K(s) g_{N}(s) f_{N}^{*}(s) d s,
$$

which by (4.3) and (4.17) becomes

$$
-\sum y_{n} g_{n}=\frac{1}{2 \pi i} \int_{L^{\prime}}\left(K_{+}(u)-\frac{1}{K_{-}(u)}\right) g(u) f^{*}(u) d u \text {. }
$$


Also for $f^{*}(s)$ satisfying (4.18) and any $f(s)$ which is regular in the strip and $O\left(t^{A}\right)$ for $t \sim \pm \infty$ we have, by commuting a double integral,

$$
\begin{aligned}
\int_{L}\left(K_{+}(u)-\frac{1}{K_{-}(u)}\right) \frac{f^{*}(u) f(u) d u}{K_{+}(u)}=-\frac{1}{2 \pi i} \int_{L}\left(K_{+}(s)-\frac{1}{K_{-}(s)}\right) f^{*}(s) d s \\
\cdot \int_{L}\left(K_{-}(u)-\frac{1}{K_{+}(u)}\right)\left(\frac{1}{s-u}+\eta_{N}(1-u+s)\right) f(u) d u .
\end{aligned}
$$

On the left we can replace the path $L$ by $L^{\prime}$ and on the right interchange the dummies $u, s$. Hence, combining this result with (4.20) and referring to the definition (4.5) of $F(s)$,

$$
\sum y_{n} g_{n}=\frac{1}{2 \pi i} \int_{L^{\prime}}\left(K_{+}(u)-\frac{1}{K_{-}(u)}\right) f^{*}(u) F(u) d u,
$$

whence substitution for $f^{*}(u)$ from (4.19) gives

$$
\begin{aligned}
2 \pi i \sum y_{n} g_{n}= & \int_{L^{\prime}}\left(K_{+}(u)-\frac{1}{K_{-}(u)}\right) P_{\mu^{\prime}-1}(u) F(u) d u \\
& -\int_{L^{\prime}}\left(K_{+}(z)-\frac{1}{K_{-}(z)}\right) \frac{f^{*}(z) d z}{2 \pi i} Q
\end{aligned}
$$

where

$$
\begin{aligned}
Q= & \int_{L^{\cdot}}\left(K_{+}(u)-\frac{1}{K_{-}(u)}\right) \frac{\eta_{N}(1-u+z) F(u) d u}{K_{+}(u)} \\
& -\int_{L}\left(K_{-}(s)-\frac{1}{K_{+}(s)}\right) \eta_{N}(1-s+z) d s \int_{L^{\prime}}\left(K_{+}(u)-\frac{1}{K_{-}(u)}\right) \frac{F(u) d u}{2 \pi i(s-u)} .
\end{aligned}
$$

Suppose now that, for the given $\left\{g_{n}\right\}, f(s)$ is the solution of (4.14); then by $\S 4.7 F(u)$ is a polynomial of degree $\mu^{\prime}-1$, so $K_{+}(u) F(u), F(u) / K_{-}(u)$ are $O\left(u^{-1}\right)$ in the right and left half-planes, respectively. In (4.21) the inner integral in the last term equals $F(s) / K_{-}(s)$, and so, $Q=0$. Hence

$$
\begin{aligned}
2 \pi i \sum y_{n} g_{n} & =\int_{L^{\prime}}\left(K_{+}(u)-\frac{1}{K_{-}(u)}\right) P_{\mu^{\prime}-1}(u) F(u) d u \\
& =-\int_{L^{\prime}}\left(K_{+}(s)-\frac{1}{K_{-}(s)}\right) P_{\mu^{\prime}-1}(s) d s \\
& \cdot\left\{g(s)-\int_{L}\left(K_{-}(z)-\frac{1}{K_{+}(z)}\right) \frac{\eta_{N}(1-s+z) f(z) d z}{2 \pi i}\right\}
\end{aligned}
$$

by (4.11). By (4.15) the vanishing of the right hand member is the consistency condition, so its equivalence to $\sum y_{n} g_{n}=0$ is established. 
4.9. Implications regarding the system (1.1). These follow immediately by combining the results of $\S \S 4.6-4.8$ with Theorem 1 ( $\$ 3.2$ ). The band of regularity (in general $0<\sigma<1$ ) of $1+K(s)$ is divided into strips $S$ by lines $\sigma=$ const. through the zeros of this function. If any such $S=S(\alpha, \beta)$ has zeros $\alpha, \beta$ on its left and right boundaries, respectively, and if the index of the strip is $\mu$, we have

THEOREM 2. For the system (1.1), in which $N$ is sufficiently large and $\left\{g_{n}\right\}$ is in $D_{\alpha}$, there is in association with any strip $S(\alpha, \beta)$ of index $\mu$

(i) if $\mu=0$, one solution $\left\{x_{n}\right\}$ in $D_{\alpha}$;

(ii) if $\mu>0$, a general solution in $D_{a}$ depending linearly on $\mu$ arbitrary constants, the coefficients of these constants giving linearly independent solutions of the homogeneous system (1.1) with $g_{n}=0$;

(iii) if $\mu<0$, one solution in $D_{\alpha}$, provided only that $\left\{g_{n}\right\}$ is orthogonal to each of the $(-\mu)$ linearly independent solutions in $D_{1-\beta}$ of the homogeneous system adjoint to (1.1).

(iv) The totality of solutions thus associated with any one of the strips $S$ is associated with the $S$ that is furthest to the right.

(iv) is true because a solution in $D_{\alpha}$ is automatically in any $D_{\alpha^{\prime}}$ for which $\operatorname{Re} \alpha^{\prime}>\operatorname{Re} \alpha$.

The consistency of the preceding statements may be seen also by consideration of the conditions that a solution in $D_{\alpha^{\prime}}$ (where the strip $S^{\prime}$ to the right of $\alpha^{\prime}$ has positive index $\mu$ ) be actually in $D_{\alpha}$ with $\operatorname{Re} \alpha<\operatorname{Re} \alpha^{\prime}$. These conditions are (a) that the associated $f_{N}(s)$ be regular at $\alpha^{\prime}$, or at the several $\alpha^{\prime}$ which may lie on the left boundary of $S^{\prime}$, and (b) that $\left\{g_{n}\right\}$ be in $D_{\alpha}$. The condition (a) is equivalent to the vanishing of $(1+K(u)) f_{N}(u)$ at $\alpha^{\prime}$, and this provides one relation between the arbitrary constants involved in $f_{N}(u)$ for each $\alpha^{\prime}$ on the boundary. Thus the number of solutions in $D_{\alpha}$ decreases stepwise as $\operatorname{Re} \alpha$ decreases. For passage from a strip with $\mu=0$ to one with $\mu<0$ no arbitrary constants remain in $f_{N}(s)$, and regularity at the zeros on the common boundary is secured only by the imposition of restrictions on the $g_{n}$.

At the heuristic level we may note that, for any solution $\left\{x_{n}\right\}$ of (1.1) in the ordinary sense, $\sum k(m, n) x_{n}$ must converge for every $m$; and since, for fixed $m, k(m, n)=O\left(n^{-1}\right)$ for $n \sim \infty$ it seems that $\sum x_{n} n^{-1}$ must converge. Then, unless $\sum x_{n} n^{-s}$ has $\sigma=1$ as its abscissa of convergence, $\left\{x_{n}\right\}$ is in some $D_{\alpha}$ with $\alpha<1$. Thus the preceding theory covers all solutions except possibly 'marginal' ones.

4.10. The integral equation

$$
x(m)+\int_{N}^{\infty} k(m, n) x(n) d n=g(m), \quad m \geqq N>0 .
$$

is the continuous analogue of (1.1). From the homogeneity property (1.2) 
of the kernel, it is equivalent to equations in which the range of integration is $(1, \infty)$ or $(0,1)$, by evident changes of variable. A particular example of the latter form was solved by A. C. Dixon [5], using ingenious adhoccery, or more compactly by Titchmarsh [6].

The methods of this paper lead directly to its solution. The analogues of (3.2), (3.3), (3.5) are

$$
\begin{gathered}
x(m)+\frac{1}{2 \pi i} \int_{c-i \infty}^{c+i \infty} K(s) m^{s-1} f_{N}(s) d s=g(m), \quad \int_{N}^{\infty} x(m) m^{-u} d m=f_{N}(u), \\
Z_{N}(z)=\int_{N}^{\infty} n^{-z} d n=\frac{N^{1-z}}{z-1}, \quad \operatorname{Re} z>1
\end{gathered}
$$

and the analogue of (3.4) is, for $\operatorname{Re} u>c$,

$$
f_{N}(u)+\frac{1}{2 \pi i} \int_{c-i \infty}^{c+i \infty} \frac{K(s) N^{s-u} f_{N}(s) d s}{u-s}=\int_{N}^{\infty} g(n) n^{-u} d n=G_{N}(u)
$$

By

$$
K_{+}(s) f_{N}(s)=N^{-s} f(s), \quad G_{N}(s)=N^{-s} G(s)
$$

this reduces to the form (4.4) with $\eta_{N}=0$, so by (4.14), (4.15) the solution is

$$
f(u)=\frac{G(u)}{K_{-}(u)}+\frac{1}{2 \pi i} \int_{L^{\prime}}\left(K_{+}(s)-\frac{1}{K_{-}(s)}\right) \frac{G(s) d s}{s-u}+ \begin{cases}0 & \text { if } \mu \leqq 0 \\ P_{\mu-1}(u) & \text { if } \mu>0,\end{cases}
$$

with the consistency condition in the case $\mu=-\mu^{\prime}<0$

$$
\int_{L^{\prime}}\left(K_{+}(s)-\frac{1}{K_{-}(s)}\right) P_{\mu^{\prime}-1}(s) G(s) d s=0 .
$$

(4.26) gives all the solutions associated with a strip of index $\mu$, under the condition that the integral defining $G_{N}(u)$ in (4 24) converges for $u$ in the strip. By taking the solutions thus associated with the furthest strip to the right in the band of regularity of $K(s)$ we get the totality of solutions for which $\int_{N}^{\infty} x(m) m^{-u} d m$ converges for some $u$ in this band.

\section{Iterative solution of the integral equation (4.14)}

5.1. Estimates for $\eta_{N}(s)$. We require estimates depending explicitly on $N$. Writing $N-\frac{1}{2}=x$ we have by the definition (4.1)

$$
\eta_{N}(s)=x^{s-1} \zeta_{N}(s)-\frac{1}{s-1}
$$

where (cf. [7]) 


$$
\begin{aligned}
\zeta_{N}(s) & =\sum_{N}^{\infty} n^{-s}=-\frac{1}{2 i} \int_{x-i \infty}^{x+i \infty} z^{-s} \cot \pi z d z \\
& =\frac{x^{1-s}}{s-1}-\frac{1}{2 i} \int_{x-i \infty}^{x}(\cot \pi z-i) z^{-s} d z-\frac{1}{2 i} \int_{x}^{x+i \infty}(\cot \pi z+i) z^{-s} d z,
\end{aligned}
$$

with the latter formula holding for all $s$. The substitutions $z=x \pm i v$ thence give

(5.2) $x \eta_{N}(s)=-\int_{0}^{\infty} \frac{2 \exp \left(-\frac{1}{2} s \log \left(1+v^{2} / x^{2}\right)\right) \sin (s \arctan v / x) d v}{e^{2 \pi v}+1}$;

and here

$$
\left|\sin \left(s \arctan \frac{v}{x}\right)\right| \leqq \sinh \frac{|s| v}{x}<\frac{|s| v}{x} \exp \left(\frac{|s| v}{x}\right) .
$$

5.11. First let $|s| \leqq \pi x$. Then for $\sigma \geqq 0$

(5.3) $\left|x \eta_{N}(s)\right|<2 \int_{0}^{\infty} \frac{|s| v}{x} \exp \left(-2 \pi v+\frac{|s| v}{x}\right) d v \leqq \frac{2|s|}{x} \int_{0}^{\infty} v e^{-\pi v} d v=\frac{2|s|}{\pi^{2} x}$.

For $\sigma=-\sigma^{\prime}<0$ we use

$$
\log \left(1+\frac{v^{2}}{x^{2}}\right) \leqq \begin{cases}v^{2} / x^{2} & \text { if } v \leqq x \\ \log \left(2 v^{2} / x^{2}\right) & \text { if } v \geqq x .\end{cases}
$$

Thus if $v \leqq x$ the modulus of the integrand in (5.2) is less than

$$
2 e^{-2 \pi v} \frac{|s| v}{x} \exp \left(\frac{\sigma^{\prime} v^{2}}{2 x^{2}}+\frac{|s| v}{x}\right) \leqq \frac{2|s| v}{x} \exp \left(-\frac{1}{2} \pi v\right)
$$

(since $|s| \leqq \pi x$ and $\sigma^{\prime} v \leqq|s| x \leqq \pi x^{2}$ ); while for $v \geqq x$ it is less than

$$
2 e^{-2 \pi v}\left(\frac{\sqrt{ } 2 v}{x}\right)^{\sigma^{\prime}} \frac{|s| v}{x} \exp \frac{|s| v}{x} \leqq \frac{2|s|}{x}\left(\frac{\sqrt{ } 2}{x}\right)^{\sigma^{\prime}} v^{1+\sigma^{\prime}} e^{-\pi v} \text {. }
$$

Hence for $\sigma=-\sigma^{\prime}<0$

$$
\begin{aligned}
\left|x \eta_{N}(s)\right| & <\frac{2|s|}{x} \int_{0}^{\infty} e^{-\pi v / 2} v d v+\frac{2|s|}{x}\left(\frac{\sqrt{ } 2}{x}\right)^{\sigma^{\prime}} \int_{0}^{\infty} e^{-\pi v} v^{1+\sigma^{\prime}} d v \\
& =\frac{8|s|}{\pi^{2} x}+\frac{2|s|}{x}\left(\frac{\sqrt{ } 2}{\pi x}\right)^{\sigma^{\prime}} \frac{\Gamma\left(2+\sigma^{\prime}\right)}{\pi^{2}} .
\end{aligned}
$$

Here, by Stirling's formula,

$$
\begin{aligned}
\left(\frac{\sqrt{ } 2}{\pi x}\right)^{\sigma^{\prime}} \Gamma\left(2+\sigma^{\prime}\right) & <3\left(\frac{\sqrt{ } 2 \sigma^{\prime}}{e \pi x}\right)^{\sigma^{\prime}}\left(1+\sigma^{\prime}\right)^{\frac{3}{2}} \\
& \leqq 3\left(\frac{\sqrt{ } 2}{e}\right)^{\sigma^{\prime}}\left(1+\sigma^{\prime}\right)^{\frac{3}{2}} \leqq \frac{3}{\sqrt{ }(2 e)}\left(\frac{3}{2 \log (e / \sqrt{ } 2)}\right)^{\frac{3}{2}},
\end{aligned}
$$


so finally if $|s| \leqq \pi x$

$$
\left|\eta_{N}(s)\right| \leqq \frac{1}{4} A|s| x^{-2} \leqq A|s| N^{-2}
$$

where $A$ is an absolute constant.

5.12. For $|s|>\pi x$ we shall restrict attention to estimates uniformly valid for $-H \leqq \sigma \leqq H$, where $H$ is an absolute but arbitrary constant. These are derived from the approximate functional equation for the zeta function [7], which gives, for $|t| \geqq K>0$ and hence for $|t| \geqq \frac{1}{2} \pi x$,

$$
\zeta_{N}(s)=\chi \sum_{n<y} n^{s-1}+O\left(x^{-\sigma}\right)+O\left(y^{\sigma-1}|t|^{\frac{1}{2}-\sigma}\right),
$$

where $y$ is defined by $2 \pi x y=|t|$ and $\chi=2^{8} \pi^{8-1} \sin \frac{1}{2} \pi s \Gamma(1-s)=O\left(|t|^{\frac{1}{2}-\sigma}\right)$. Hence by (5.1)

$$
x \eta_{N}(s)=x^{s} \zeta_{N}(s)-\frac{x}{s-1}=O\left(x^{\sigma}|t|^{1-\sigma}\right) \sum_{n<v} n^{\sigma-1}+O(1)+O\left(x|t|^{-\frac{1}{2}}\right) .
$$

By using an elementary estimate for $\sum n^{\sigma-1}$ we obtain

$$
\begin{aligned}
& x \eta_{N}(s)=O\left(|t|^{\frac{1}{2}}\right)+O\left(x|t|^{-\frac{1}{2}}\right), \quad 0<\sigma_{0} \leqq \sigma \leqq H, \\
& x \eta_{N}(s)=O\left(|t|^{\frac{1}{2}}\left|\frac{t}{x}\right|^{-\sigma}\right)+O\left(x|t|^{-\frac{1}{2}}\right), \quad-H \leqq \sigma \leqq-\sigma_{0}<0 ;
\end{aligned}
$$

for $-\sigma_{0} \leqq \sigma \leqq \sigma_{0}$, a factor $\log |t| x \mid$ is to be adjoined to the first term on the right.

In view of $\left(\mathbf{5 . 4 )}\right.$ these estimates need be used only for $|t| x \mid \geqq \frac{1}{2} \pi$, so we can simplify (and weaken) them by inserting positive powers of $|t| x \mid$ on the right. Hence (5.5) gives $x^{2} \eta_{N}(s)=O\left(|t|^{\frac{8}{2}}\right)$, and in combination with (5.4) we have

$$
\left|\eta_{N}(s)\right| \leqq A_{1}\left(1+|t|^{\frac{3}{2}}\right) N^{-2}, \quad 0<\sigma_{0} \leqq \sigma \leqq H, \text { all } t,
$$

for some positive constant $A_{1}$.

5.2. Iterative solution of (4.14). We abbreviate the equation as

$$
f(u)-\int_{-\infty}^{\infty} \phi_{1}(u, z) f(z) d t-\int_{-\infty}^{\infty} \phi_{2}(u, z) f(z) d t=h(u)
$$

when $t=\operatorname{Im} z$ and

$$
\begin{gathered}
\phi_{1}(u, z)=-\left(K_{-}(z)-\frac{1}{K_{+}(z)}\right) \frac{\eta_{N}(1+u-z)}{2 \pi K_{-}(u)}, \\
\phi_{2}(u, z)=-\left(K_{-}(z)-\frac{1}{K_{+}(z)}\right) \int_{L^{\prime}}\left(K_{+}(s)-\frac{1}{K_{-}(s)}\right) \frac{\eta_{N}(1+s-z) d s}{4 \pi^{2} i(s-u)},
\end{gathered}
$$

and $h(u)$ is the function on the right of (4.14). We take $u$ to lie on the same path $L$ as $z$, and write $v=\operatorname{Im} u$, so taking $\sigma=1$ in (5.7) 


$$
\left|\eta_{N}(1+u-z)\right| \leqq A_{1}\left(1+2|v|^{\frac{3}{2}}+2|t|^{\frac{1}{2}}\right) N^{-2} .
$$

In (5.9) we have, from (2.8), (1.7) and (1.17), for absolute positive constants $A_{2}, A_{3}$, and $\lambda^{\prime}<\lambda$

$$
\left|K_{-}(z)-\frac{1}{K_{+}(z)}\right| \leqq A_{2} e^{-\lambda^{\prime}|t|}, \quad\left|\frac{1}{K_{-}(u)}\right| \leqq A_{3}\left(1+|v|^{\mu}\right)
$$

so

$$
\left|\phi_{1}(u, z)\right| \leqq \frac{A_{1} A_{2} A_{3}}{2 \pi N^{2}}\left(1+|v|^{\mu}\right)\left(1+2|v|^{\frac{z}{2}}+2|t|^{\frac{3}{2}}\right) e^{-\lambda^{\prime}|t|} .
$$

In (5.10) the integration is along a path $L^{\prime}$ to the right of $L$, so $|s-u|$ exceeds the positive constant $\operatorname{Re}(s-u)$. For $\eta_{N}(1+s-z)$ there is an inequality of the form (5.11), and for $\left|K_{+}(s)-1 / K_{-}(s)\right|$ one of the form $(5.12)_{1}$. Hence we have the form

$$
\left|\phi_{2}(u, z)\right| \leqq A_{4} N^{-2}\left(1+2|t|^{2}\right) e^{-\lambda^{\prime}|t|}
$$

with $A_{4}$ an absolute constant.

For $h(u)$ in $(5.8)$ we have

$$
|h(u)| \leqq B\left(1+|v|^{\rho}\right)
$$

where $\rho$ is some positive constant, and $B$ a constant whose dependence on $N$ is not relevant.

For the solution of (5.8) we define an iterative sequence by

$$
f_{n+1}(u)=h(u)+\int \phi_{1}(u, z) f_{n}(z) d t+\int \phi_{2}(u, z) f_{n}(z) d t, \quad n=0,1,2, \cdots
$$

starting with $f_{0}(u)=h(u)$. Then, writing

$$
\chi_{0}(u)=f_{0}(u)=h(u), \quad \chi_{n+1}(u)=f_{n+1}(u)-f_{n}(u), \quad n \geqq 0
$$

we have

$$
\left|\chi_{n+1}(u)\right| \leqq \int\left|\phi_{1}(u, z) \chi_{n}(z)\right| d t+\int\left|\phi_{2}(u, z) \chi_{n}(z)\right| d t .
$$

From the form in which $|v|$ is involved in (5.13), (5.14) it is seen that a hypothesis which is suitable for inductive verification is

$$
\left|\chi_{n}(z)\right| \leqq M_{n}\left(1+|t|^{\mu}\right)\left(1+|t|^{\frac{3}{2}}\right)
$$

with $M_{n}$ some constant. On substitution of this, along with (5.13), (5.14) in (5.17) the integrals converge because of the exponential factor and we obtain the form

$$
\left|\chi_{n+1}(u)\right| \leqq N^{-2} M_{n}\left\{\left(1+|v|^{\mu}\right)\left(A_{5}+A_{6}|v|^{\frac{3}{2}}\right)+A_{7}\right\}
$$

with $A_{5}, A_{6}, A_{7}$ absolute constants. Hence $\chi_{n+1}(u)$ satisfies an inequality of the form (5.18), with 


$$
M_{n+1} / M_{n}=N^{-2} \max \left(A_{5}+A_{7}, A_{6}\right)
$$

and we shall have $M_{n+1} / M_{n} \leqq q<1$ if

$$
N^{2} \geqq q^{-1} \max \left(A_{5}+A_{7}, A_{6}\right), \quad 0<q<1 .
$$

To start the induction we have on the right of (5.17) $\chi_{0}(z)=f_{0}(z)=h(z)$, and by (5.15) $\left|\chi_{0}(z)\right| \leqq B\left(1+|t|^{\rho}\right)$. The difference in form between this and (5.18) does not disturb the form (5.19) for $\chi_{1}(u)$; only the values of the constants $A_{5}, A_{6}, A_{7}$ are altered. Hence if $N$ is large enough to satisfy (5.20) we have

$$
\left|\chi_{n}(z)\right| \leqq q^{n} A_{8} B\left(1+|t|^{\mu}\right)\left(1+|t|^{\frac{3}{2}}\right), \quad n=1,2, \cdots .
$$

By standard arguments it follows that the sequence $f_{n}(z)$ converges uniformly over any bounded range of $\operatorname{Im} z$, that the limit function $f(z)$ satisfies (4.14), and that this is the only solution which is continuous and $O\left(|\operatorname{Im} z|^{A}\right)$ for any constant $A$.

All this holds for $u$ on the path $L$ in (4.14). For $u$ not on $L$, but near it, we now use (4.14) to define $f(u)$, and the regularity of the integrands secures that this gives an analytic function which is the continuation of $f(u)$ from $L$. Indeed, the equation shows that $f(u)$ can be continued to all points to the left of $L$ where $g(u)$ is regular. Finally, for $u$ on $L$ we can in (4.14) replace the integrals along $L^{\prime}$ by integrals along a path to the left of $L$, which gives a form in which the terms $\eta_{N}(1+u-z) / K_{-}(u), g(u) / K_{-}(u)$ are replaced by $\eta_{N}(1+u-z) K_{+}(u), g(u) K_{+}(u)$; and hence $f(u)$ can be continued indefinitely to the right of $L$.

\section{The general system (1.1)}

6.1. We now suppose that in (1.1) $N$ is not large enough - in relation to $k(m, n)$ - for solubility of (4.14) by iteration, so that this equation may present the 'eigen-alternative' in which the homogeneous form has non-trivial solutions. The theory regarding (1.1) may be based on the Fredholm theorems, but this treatment cannot be quite straightforward because, if $\mu>0$, a non-homogeneous form of (4.14) is associated with the homogeneous form of (1.1). It is preferable therefore to make a direct investigation of (1.1) by partitioning this system. We choose an $N^{\prime}$ large enough for Theorem 2 to apply to the solution for $x_{N^{\prime}}, x_{N^{\prime}+1}, \cdots$ of the sub-set of equations for which $m \geqq N^{\prime}$. The solution involves $x_{N}, \cdots x_{N^{\prime}-1}$ as parameters, and its substitution in the earlier equations reduces these to a finite set in these parameters. The reduced set may present the 'eigenalternative', but this is then an algebraic phenomenon, quite distinct from the transcendental phenomena associated with the index. Hence we shall prove 
THEOREM 3. Let $\alpha, \beta$ be respectively to the left and right of a zero-free strip of regularity of $1+K(s)$ whose index is $\mu$. Then

(i) the number of linearly independent solutions in $D_{\alpha}$ of the homogeneous system (1.1) is $\mu+p$ when $\mu>0$ or $p$ when $\mu \leqq 0$, where $p$ is a non-negative integer;

(ii) the number of such solutions exceeds by $\mu$, exactly, the number of linearly independent solutions in $D_{1-\beta}$ of the homogeneous adjoint system,

(iii) when $\left\{g_{n}\right\}$ is in $D_{\alpha}$, the non-homogeneous system is soluble in $D_{\alpha}$ if and only if $\left\{g_{n}\right\}$ is orthogonal to all solutions in $D_{1-\beta}$ of the homogeneous adjoint system.

To prove this it is convenient to use matrix notation, taking $\boldsymbol{x}=\left\{x_{N}, x_{N+1}, \cdots\right)$ and $\boldsymbol{g}=\left\{g_{N}, g_{N+1}, \cdots\right\}$ as column vectors. For the adjoint system we take $\boldsymbol{y}=\left(y_{N}, y_{N+1}, \cdots\right)$ as a row vector. Choosing a large enough $N^{\prime}$ we partition these vectors:

$$
x=\left(\begin{array}{l}
x^{\prime} \\
x^{\prime \prime}
\end{array}\right), \quad g=\left(\begin{array}{l}
g^{\prime} \\
g^{\prime \prime}
\end{array}\right), \quad y=\left(y^{\prime}, y^{\prime \prime}\right)
$$

where $\boldsymbol{x}^{\prime}=\left\{x_{N}, \cdots x_{N^{\prime}-1}\right\}, \boldsymbol{x}^{\prime \prime}=\left\{x_{N^{\prime}}, x_{N^{\prime}+1}, \cdots\right\}$ etc. With a corresponding partition

$$
\left(\delta_{m n}+k(m, n)\right)=\left(\begin{array}{ll}
\boldsymbol{A} & \boldsymbol{B} \\
\boldsymbol{C} & \boldsymbol{D}
\end{array}\right)
$$

(1.1) splits into

$$
\begin{aligned}
A x^{\prime}+B x^{\prime \prime} & =g^{\prime} \\
D x^{\prime \prime} & =g^{\prime \prime}-C_{X^{\prime}},
\end{aligned}
$$

and the homogeneous adjoint system splits into

$$
\begin{aligned}
\boldsymbol{y}^{\prime} \boldsymbol{A}+\boldsymbol{y}^{\prime \prime} \boldsymbol{C} & =0 \\
\boldsymbol{y}^{\prime \prime} D & =-\boldsymbol{y}^{\prime} \boldsymbol{B} .
\end{aligned}
$$

In the manipulations that follow, the operations that involve infinite matrices are validated by $\S 3.4$; by Lemma 2 the series which define elements of a product matrix converge, and by Lemma 3, applied to each element of a triple product, such a product obeys the associative law. For example, in $(6.13)_{1}$ we have the case of $y$-finite summation, and in $(6.13)_{2}$ the case referred to in the Note to Lemma 3 where in the notation of that Lemma, each column of $\boldsymbol{H}$ satisfies a system with $g_{m}=0$ and $\boldsymbol{y}^{\prime \prime}$ satisfies a system (6.6) with

$$
h_{n}=-\sum_{m=N}^{N^{\prime}-1} y_{m}^{\prime} k(m, n) .
$$


6.2. The case $\mu \geqq 0$. By Theorem 2 the homogeneous system $D x^{\prime \prime}=0$ has $\mu$ linearly independent solutions in $D_{\alpha}$ which can be set out as a matrix $\boldsymbol{H}$ of $\mu$ columns and infinitely many rows, and the most general solution of $(6.4)^{5}$ in $D_{\alpha}$ has the form

$$
\boldsymbol{x}^{\prime \prime}=\xi^{\prime \prime}-E x^{\prime}+H \lambda
$$

where $\lambda$ is a column vector of $\mu$ arbitrary components. The conditions that (6.7) satisfies (6.4) are

$$
D \xi^{\prime \prime}=g^{\prime \prime}, \quad D E=C, \quad D H=0 .
$$

By substitution of (6.7) into (6.3) this becomes

$$
(\boldsymbol{A}-\mathrm{BE}) \boldsymbol{x}^{\prime}+\boldsymbol{B H} \boldsymbol{\lambda}=\boldsymbol{g}^{\prime}-B \boldsymbol{\xi}^{\prime \prime}
$$

This is a set of $N^{\prime}-N$ scalar equations, for whose satisfaction the components of $\lambda$ as well as those of $\boldsymbol{x}^{\prime}$ are disposable, so the critical thing is the rank of the matrix $(A-B E, B H)$ of $N^{\prime}-N$ rows and $N^{\prime}-N+\mu$ columns. If this rank is $N^{\prime}-N$ the set is soluble for $N^{\prime}-N$ of the components of $\boldsymbol{x}^{\prime}, \lambda$, without restriction on $\boldsymbol{g}^{\prime}$, with the remaining components remaining arbitrary, and the full system has a solution $\left\{\boldsymbol{x}^{\prime}, \boldsymbol{x}^{\prime \prime}\right\}$ in $D_{\alpha}$ depending linearly on $\mu$ arbitrary constants. But if the rank is $N^{\prime}-N-p$ the homogeneous system (6.9) with $\boldsymbol{g}^{\prime}=\xi^{\prime \prime}=0$ has $\mu+p$ linearly independent solutions (leading via (6.7) to a like number of solutions of the full homogeneous system), and the non-homogeneous (6.9) is soluble if and only if $\boldsymbol{g}^{\prime}-\boldsymbol{B} \boldsymbol{\xi}^{\prime \prime}$ is orthogonal to every solution $\boldsymbol{y}^{\prime}$ of the adjoint system

$$
\begin{array}{r}
\boldsymbol{y}^{\prime}(\boldsymbol{A}-\boldsymbol{B E})=0 \\
\boldsymbol{y}^{\prime}(\boldsymbol{B H})=0 .
\end{array}
$$

This condition is

$$
\boldsymbol{y}^{\prime}\left(\boldsymbol{g}^{\prime}-B \xi^{\prime \prime}\right)=0 .
$$

Considering now the solution of the homogeneous adjoint system, post multiplication of (6.6) by $\boldsymbol{H}$ gives, in virtue of $(6.8)_{3}, \boldsymbol{y}^{\prime}(\boldsymbol{B H})=\mathbf{0}$ as a necessary condition for its solubility for a $y^{\prime \prime}$ in $D_{1-\beta}$; and by Theorem 2 this condition is sufficient. Here we have used the associative properties

$$
\left(\boldsymbol{y}^{\prime} B\right) \boldsymbol{H}=\boldsymbol{y}^{\prime}(B H), \quad\left(\boldsymbol{y}^{\prime \prime} D\right) \boldsymbol{H}=\boldsymbol{y}^{\prime \prime}(\boldsymbol{D H}) .
$$

By use of $(6.8)_{2}$ and similar associations the elimination of $y^{\prime \prime}$ from (6.5) and (6.6) gives $y^{\prime}(A-B E)=0$. Thus $y^{\prime}$ must satisfy (6.10) and (6.11); and conversely any solution of these leads to a solution of (6.5) and (6.6). By a well known theorem of algebra, (6.10) and (6.11) have just $p$ linearly

\footnotetext{
5 Where $\boldsymbol{g}^{\prime \prime}$ (by hypothesis) and each column of $\mathcal{C}_{\boldsymbol{x}^{\prime}}$ (direct proof via (1.4)) is in $D_{\alpha}$.
} 
independent solutions $\boldsymbol{y}^{\prime}$ when the rank of the matrix $(\boldsymbol{A}-\boldsymbol{B E}, \boldsymbol{B H})$ is $N^{\prime}-N-p$.

Thus we have established so much of (i), (ii) of Theorem 3 as belongs to the case $\mu \geqq 0$.

As regards (iii) we observe that from (6.6) and (6.8) we have

so

$$
\boldsymbol{y}^{\prime} \boldsymbol{B}^{\prime \prime}=-\boldsymbol{y}^{\prime \prime} D \xi^{\prime \prime}=-\boldsymbol{y}^{\prime \prime} \boldsymbol{g}^{\prime \prime}
$$

$$
\boldsymbol{y}^{\prime}\left(\boldsymbol{g}^{\prime}-B \xi^{\prime \prime}\right)=\boldsymbol{y}^{\prime} \boldsymbol{g}^{\prime}+\boldsymbol{y}^{\prime \prime} \boldsymbol{g}^{\prime \prime} \text {. }
$$

Hence, for any admissible $y^{\prime}, y^{\prime \prime}$ the condition (6.12) is equivalent to $\boldsymbol{y}^{\prime} \boldsymbol{g}^{\prime}+\boldsymbol{y}^{\prime \prime} \boldsymbol{g}^{\prime \prime}=0$, i.e. to the orthogonality of $\left\{\boldsymbol{g}^{\prime}, \boldsymbol{g}^{\prime \prime}\right\}$ to any solution $\left(y^{\prime}, y^{\prime \prime}\right)$ of the full homogeneous adjoint system. Since (6.12) is just the condition for solubility via (6.9) of the direct system (6.3), (6.4), Theorem 3 (iii) is established, for the case $\mu \geqq 0$.

6.3. The case $\mu=-\mu^{\prime}<0$ is similarly handled, and we omit the proof. The treatment starts with an appeal to Theorem 2 , whereby (i) $y^{\prime \prime} D=0$ has a set of $\mu^{\prime}$ solutions which can be combined in the form $y^{\prime \prime}=\lambda J$, with $\lambda$ a row vector with $\mu^{\prime}$ arbitrary components and $J$ a matrix of $\mu^{\prime}$ rows and $\infty$ columns; and (ii) (6.4) is soluble for $x^{\prime \prime}$ if only $J\left(g^{\prime \prime}-C_{x^{\prime}}\right)=0$.

6.4. Concerning inverse matrices. In the case of Theorem 3 where $\mu \geqq 0$ and $p=0$ the system

$$
\boldsymbol{M} \boldsymbol{x}_{\rho}=\left(\delta_{m n}+k(m, n)\right) \boldsymbol{x}_{\rho}=\boldsymbol{g}_{\rho}=\left\{\left(\delta_{m \rho}\right)\right\}
$$

is soluble in $D_{\alpha}$, and any solution $x_{\rho}$ gives the $\rho$ th column of a matrix $X$ which is a right inverse in $D_{\alpha}$ of $\boldsymbol{M}: \boldsymbol{M X}=\boldsymbol{I}$. If $\mu=0$ there is only one such $X$, but if $\mu>0$ there are an infinity of them. If $\mu=0$ there is also a left inverse $\boldsymbol{Y}$ of $\boldsymbol{M}$ whose rows are in $D_{1-\beta}$ and are obtained by solving systems adjoint to (6.14): $Y M=I$; but if $\mu>0$ there can be no such inverse in $D_{1-\beta}$ since its multiplication into (6.14) would furnish for this system a unique solution. These circumstances resemble those that can arise for bounded matrices over Hilbert space [8].

In the case $\mu=0$, Lemma 3 gives $\boldsymbol{Y}(\boldsymbol{M} \boldsymbol{X})=(\boldsymbol{Y M}) \boldsymbol{X}$, since the second alternatives of hypotheses (i) and (ii) are satisfied by the particular $\boldsymbol{g}_{\rho}$ in (6.14) and its adjoint. Hence

$$
\boldsymbol{Y}=\boldsymbol{Y I}=\boldsymbol{Y}(\boldsymbol{M} \boldsymbol{X})=(\boldsymbol{Y M}) \boldsymbol{X}=\boldsymbol{I} \boldsymbol{X}=\boldsymbol{X} .
$$

Hence in the general case where there are several strips, of which an interior one $S(\alpha, \beta)$ has index 0 , we have in the case $p=0$ - which always holds when $N$ is sufficiently large - there is a matrix $\boldsymbol{M}^{-1}$ with rows in $D_{1-\beta}$ and columns in $D_{\alpha}$, which is both a right- and left-inverse for $\boldsymbol{M}$; this is also one of the right [left] inverses associated with strips to the right [left] of $S(\alpha, \beta)$. 
When any possible solution of (1.1) that is relevant to a given context is known, from that context, to be unique, Theorem 3 shows that the solution cannot be associated only with a strip where $\mu>0$; and if there is a strip with $\mu=0$ we must have the case $p=0$, wherein $M$ has identical right and left inverses. The solubility of (1.1) will then follow if $\left(g_{n}\right)$ lies in a $D_{a}$ with $\alpha$ to the left of the strip. Thus, with the stated proviso, uniqueness implies existence for the solution of (1.1).

\section{Asymptotic formulae and procedures for approximation}

7.1. Any solution $\left\{x_{n}\right\}$ of (1.1), of the wide class which we have been considering, is in one-one correspondence with a solution $f_{N}(s)$ of the integral equation (3.4) in which the path of integration is suitably chosen. The connexion is given by (3.2), which on substitution for $f_{N}(s)$ from (4.3) and $K(s)$ from (4.2) becomes

$$
x_{m}=g_{m}-\frac{1}{2 \pi i m} \int_{c-i \infty}^{c+i \infty}\left(K_{-}(s)-\frac{1}{K_{+}(s)}\right)\left(\frac{m}{N-\frac{1}{2}}\right)^{s} f(s) d s
$$

with $f(s)$ satisfying (4.4) and (4.14), and $K_{-}(s), K_{+}(s)$ defined relative to the zero-free strip of regularity of $1+K(s)$ which contains the path $\sigma=c$. We have also to suppose that the path lies in the right half-plane in which

$$
\sum g_{n} n^{-s},=g_{N}(s)
$$

converges, which is a restriction on $\left\{g_{n}\right\}$. When there are strips of positive index, our concern will often be with that solution for which $\sum x_{n} n^{-s}$ converges most rapidly, and then the path will be chosen in the strip of least non-negative index in which (7.2) converges.

We confine attention now to the important case in which (i) $K(s)$ is meromorphic, with poles at $s=0, \pm 1, \pm 2, \cdots$ at most, and (ii) $g_{N}(s)$ has a meromorphic continuation. Then $K_{+}(s), K_{-}(s)$ are meromorphic, and by the concluding paragraph of $\S 5.2 f(s)$ is meromorphic. Hence in (7.1) we can displace the path to the left, and from the residues at the poles of the integrand that are thereby crossed we obtain an approximation to $x_{m}$ which is clearly asymptotic for $m \sim \infty-$ for given $f(s)$. The general formulae are shown in $\S 7.2$, and some examples in $\S 7.3$. When $x_{m}$ has been determined from these formulae, to adequate accuracy when $m$ is sufficiently large, the outstanding $x_{m}$ can be determined from a finite system by straight arithmetic.

An alternative procedure, which may be more efficient, is to evaluate an asymptotic formula, $\bar{x}_{m}$ say, for all values of $m$ and calculate the residual

$$
\bar{g}_{m}=g_{m}-\bar{x}_{m}-\sum_{n} k(m, n) \bar{x}_{n} \text {. }
$$


Then the exact solution $x_{m}=\bar{x}_{m}+x_{m}^{\prime}$ is to be determined from

$$
x_{m}^{\prime}+\sum_{n} k(m, n) x_{n}^{\prime}=\bar{g}_{m},
$$

which may be more favourable for approximate solution. See $\S$ 7.4. Here it is unnecessary that $\bar{x}_{m}$ be derived from an asymptotic formula; we can try with any $\bar{x}_{m}$ that can plausibly be supposed an approximation.

Finally in $\S 7.5$ we apply the calculation of residuals to the adjoint system (3.7), taking $h_{n}=\delta_{\rho n}$. An exact solution of this system, in the appropriate $D_{1-\beta}$, would give the successive rows of a left inverse for (1.1), for $\rho=N, N+1, \cdots$, and its multiplication into (1.1) would yield the explicit solution for each $x_{m}$. The exact solution of the adjoint system is of course in practice unobtainable, but there is an explicit approximation to it; the residuals from this approximation give the coefficients in a reduced system for the $x_{m}$, and the reduced system so found is no longer singular; it is of the simple type related to completely continuous bilinear forms over Hilbert space. This procedure does not provide an existence theorem, since (apparently) the passage from the original to the reduced system is not reversible. Hence it serves merely for convenient calculation of a solution which is already known to exist.

Some parts of what follows are suggestive only, but most of it (including $\S 7.5$ ) is rigorous.

7.2. Asymptotic formulae. In (7.1) the poles of the integrand are the zeros of $K_{+}(s)$, all to the left of the path, (which we now denote generically by $\alpha$ ), and the poles of $f(s)$, which by (4.14) are just the poles $\gamma$ of $g(s)$ or $g_{N}(s)$, with res. $f(s)=$ res. $g(s) / K_{-}(\gamma)$. In general the zeros $\alpha$ and poles $\gamma$ are simple, and no $\gamma$ coincides with an $\alpha$; then all the poles for (7.1) are simple, and each term in the approximation to $x_{m}$ varies as a power of $m$. If there are multiple poles they contribute logarithmic terms. In the general case, displacement of the path to the left gives

$$
\begin{aligned}
x_{m} \sim g_{m} & +\frac{1}{m} \sum_{\gamma}\left(\frac{1}{K_{+}(\gamma) K_{-}(\gamma)}-1\right) \text { res. }_{\gamma} g(s)\left(\frac{m}{N-\frac{1}{2}}\right)^{\gamma} \\
& +\frac{1}{m} \sum_{\alpha} \frac{f(\alpha)}{K_{+}^{\prime}(\alpha)}\left(\frac{m}{N-\frac{1}{2}}\right)^{\alpha}
\end{aligned}
$$

which may be interpreted either as a finite sum plus a remainder-integral (not exhibited) or as an asymptotic series.

Here $f(\alpha)$ is given implicitly by (4.14), where the integral on the left is $O\left(N^{-2}\right)$. For the integral on the right, evaluation from residues at the poles in the right half-plane is suggested, these poles being the zeros of $K_{-}(s)$, which we denote generically by $\beta$. For certain characteristic functions 
$K(s)$, at least, the integral is thus expressed as a convergent series, whence (4.14) gives

$$
f(s)=\frac{g(s)}{K_{-}(s)}+\sum_{\beta} \frac{g(\beta)}{(\beta-s) K_{-}^{\prime}(\beta)}+O\left(\frac{\mathrm{I}}{N^{2}}\right)
$$

for the 'principal case' in which $P_{\mu-1}(u)=0$. A different method for approximating to $f(\alpha)$ is shown in $\S 7.4$ (ii).

7.3. Special cases.

$$
g_{m}=m^{\gamma-1}
$$

gives

whence by $(4.3)$

$$
g_{N}(s)=\sum_{N}^{\infty} m^{\gamma-1-s}=\zeta_{N}(1+s-\gamma)
$$

$$
g(s)=\left(N-\frac{1}{2}\right)^{s} \zeta_{N}(1+s-\gamma) .
$$

This has a simple pole at $s=\gamma$, with residue $\left(N-\frac{1}{2}\right)^{\gamma}$. The middle term of (7.5) combines with the term $g_{m}$ to give

$$
x_{m} \sim \frac{m^{\gamma-1}}{1+K(\gamma)}+\frac{1}{m} \sum_{\alpha} \frac{f(\alpha)}{K_{+}^{\prime}(\alpha)}\left(\frac{m}{N-\frac{1}{2}}\right)^{\alpha}
$$

where the first term is dominant if $\gamma$ is to the right of all the zeros $\alpha$ of $K_{+}(s)$.

$$
g_{m}=k(m, v)
$$

arises as the coefficient of a component $x_{\nu}$ of the vector $x^{\prime}$ in (6.4), in which case $v<N^{\prime}$. By (1.4) this gives

$$
\begin{aligned}
&\left(N-\frac{1}{2}\right)^{-u} g(u)= g_{N}(u)=\frac{1}{2 \pi i} \int_{c-i \infty}^{c+i \infty} K(s) \nu^{-s} \zeta_{N}(1+u-s) d s, \\
&=K(u) v^{-u}+\frac{1}{2 \pi i} \int_{c^{\prime}-i \infty}^{e^{\prime}+i \infty} K(s) v^{-s} \zeta_{N}(1+u-s) d s, \\
& c<c^{\prime}<1, \quad \operatorname{Re} u<c^{\prime} .
\end{aligned}
$$

The latter form shows that $g(u)$ has as poles those of $K(u)$ to the left of its band of regularity, in general $u=0,-1,-2, \cdots$. At these points $K_{+}(u)=\infty$, and (7.5) becomes

$$
x_{m} \sim k(m, v)-\frac{1}{m} \sum_{\gamma=0,-1, \cdots} \text { res. }_{\gamma} K(s)\left(\frac{m}{v}\right)^{\gamma}+\frac{1}{m} \sum_{\alpha} \frac{f(\alpha)}{K_{+}^{\prime}(\alpha)}\left(\frac{m}{N-\frac{1}{2}}\right)^{\alpha} .
$$

For the calculation of $f(\alpha)$ via (7.6) we require $g(s)$, and for this an asymptotic formula is obtained from (7.8) by displacing the path to the left across the poles of $K(s)$ at $s=0,-1, \cdots$. 
(iii)

$$
g_{m}=\delta_{m \nu}, \quad \nu \geqq N
$$

is a form from which the general system (1.1) may be formally built by superposition; the corresponding solution $\left\{x_{m}\right\}$ gives the $\nu$ th column of a right inverse of the matrix $\left(\delta_{m n}+k(m, n)\right)$. Here

$$
g_{N}(s)=\nu^{-s}, \quad g(s)=\left(\frac{N-\frac{1}{2}}{\nu}\right)^{s},
$$

an integral function. Hence $\sum_{\gamma}$ is absent from (7.5), and substitution from (7.6) gives

$$
x_{m} \sim \sum_{\alpha} \frac{1}{m K_{+}^{\prime}(\alpha)}\left(\frac{m}{\nu}\right)^{\alpha}\left\{\frac{1}{K_{-}(\alpha)}+\sum_{\beta} \frac{1}{(\beta-\alpha) K_{-}^{\prime}(\alpha)}\left(\frac{N-\frac{1}{2}}{\nu}\right)^{\beta-\alpha}+O\left(\frac{1}{N^{2}}\right)\right\} .
$$

Since $\alpha$ runs through a sequence of values whose real parts decrease towards $-\infty$ this form can be valid only when $m / v$ is large, and since then $g_{m}=\delta_{m v}=0$ this term has been omitted from (7.11).

When $\nu$ is large and $m / v$ small, a companion approximation is obtained by substituting from (7.6) into (7.1) and splitting off the resulting term in $g(s)$, for which the path is displaced to the right across zeros $\beta$ of $K_{-}(s)$. For the remaining terms involving $\sum_{\beta}$ the path is displaced to the left across zeros $\alpha$ of $K_{+}(s)$. This gives

$$
x_{m} \sim-\sum_{\beta} \frac{1}{m K_{-}^{\prime}(\beta)}\left(\frac{m}{\nu}\right)^{\beta}\left\{\frac{1}{K_{+}(\beta)}-\sum_{\alpha} \frac{1}{(\beta-\alpha) K_{+}^{\prime}(\alpha)}\left(\frac{N-\frac{1}{2}}{m}\right)^{\beta-\alpha}+O\left(\frac{1}{N^{2}}\right)\right\},
$$

a form in which the early terms are dominant when $m / \nu$ and $\left(N-\frac{1}{2}\right) / m$ are both small.

\subsection{Residuals.}

(i) In (7.3) let us take

$$
\bar{x}_{m}=\frac{m^{\gamma-1}}{1+K(\gamma)},
$$

a form suggested by (7.7) for association with $g_{m}=m^{\gamma-1}$. Then by (1.4)

$$
\begin{aligned}
\bar{g}_{m} & =g_{m}-\bar{x}_{m}-\sum_{n} k(m, n) \bar{x}_{n} \\
& =\frac{1}{1+K(\gamma)}\left\{m^{\gamma-1} K(\gamma)-\frac{1}{2 \pi i} \int_{c-i \infty}^{c+i \infty} K(s) m^{s-1} \zeta_{N}(1+s-\gamma) d s\right\} \\
& \sim-\sum_{s=0,-1, \cdots} m^{s-1} \zeta_{N}(1+s-\gamma) \text { res. } K(s) /(1+K(\gamma)) ;
\end{aligned}
$$

the contribution from the pole of $\zeta_{N}$ at $s=\gamma$ has cancelled the term $m^{\gamma-1} K(\gamma)$. This gives a reduced system (7.4) for which $\left\{\bar{g}_{m}\right\}$ is in $D_{0}$, whereas 
by (7.13) the component $\left\{\bar{x}_{m}\right\}$ of the full solution is only in $D_{\gamma}$, where $\operatorname{Re} \gamma$ may exceed 0 .

(ii) For the case where $g_{N}(s)$ is an integral function let us adopt a form suggested by (7.5) and put

$$
\bar{x}_{m}=g_{m}+\sum_{\alpha} a_{\alpha} m^{\alpha-1}
$$

where on the right we have a sum over a finite set of $p+1$ zeros $\alpha$ of $K_{+}(s)$ to the left of the line of integration in (1.4). An easy calculation gives

$$
\begin{aligned}
\bar{x}_{m}+\sum k(m, n) \bar{x}_{n}= & g_{m}+\sum a_{\alpha} m^{\alpha-1} \\
& +\frac{1}{2 \pi i} \int_{c-i \infty}^{c+i \infty} K(s) m^{s-1}\left(g_{N}(s)+\sum_{\alpha} a_{\alpha} \zeta_{N}(1+s-\alpha)\right) d s .
\end{aligned}
$$

Since $K(s)+1=K_{+}(s) K_{-}(s)$ we have $K(\alpha)=-1$, so the residue at $s=\alpha$ is $-a_{\alpha} m^{\alpha-1}$, and the sum of these residues cancels the middle term on the right, irrespective of the values of the coefficients $a_{\alpha}$. We can then determine the $a_{\alpha}$ so that

$$
g_{N}(s)+\sum_{\alpha} a_{a} \zeta_{N}(1+s-\alpha)=0 \text { for } s=0,-1, \cdots,-p,
$$

whereby the poles of $K(s)$ at these points are cancelled. Displacement of the path across these points then gives, for $m$ large

$$
\bar{x}_{m}+\sum k(m, n) \bar{x}_{n}=g_{m}+O\left(m^{-s-2}\right) .
$$

Thus, with the $a_{\alpha}$ determined from (7.15), the residuals $\vec{g}_{m}$ in the equations of high order are small, and the reduced system (7.4) is likely to be adequately approximated by a finite system for the early $x_{m}^{\prime}$.

From (7.5) it is suggested that the solution of (7.15) should give

$$
a_{\alpha} \approx \frac{f(\alpha)\left(N-\frac{1}{2}\right)^{-\alpha}}{K_{+}^{\prime}(\alpha)},
$$

and the following 'argument' substantiates this. The $f(s)$ which corresponds - by (4.14) with $P_{\mu-1}=0$ - to an integral function $g(s)$ is everywhere regular, so reference to $(4.3)$ shows that $f_{N}(s)$ vanishes at the poles $s=0,-1, \cdots$ of $K_{+}(s)$ and has a pole at each zero $\alpha$ of $K_{+}(s)$ with residue $\left(N-\frac{1}{2}\right)^{-\alpha} f(\alpha) / K_{+}^{\prime}(\alpha)$. Now in (3.4) we can displace the path to the left and so obtain a continuation of $f_{N}(u)$ which is valid for any $u$ to the right of the displaced path. For such a $u$ this gives, from residues at the poles of $K_{+}(s) f_{N}(s)$,

$$
f_{N}(u) \sim g_{N}(u)+\sum_{\alpha} \frac{\left(N-\frac{1}{2}\right)^{-\alpha}}{K_{+}^{\prime}(\alpha)} f(\alpha) \zeta_{N}(1+u-\alpha),
$$

and the vanishing of $f_{N}(u)$ at $u=0,-1, \cdots$ gives asymptotic equations 
of the form (7.15), with coefficients as in (7.16). For a fixed $\alpha$ we can expect (7.16) to be asymptotically true for $p \sim \infty$ in (7.15).

7.5. Derivation from (1.1) of a regular system. The suggestion for what follows arises from scrutiny of the formulae (7.11), (7.12) which relate to a right inverse of $\left(\delta_{m n}+k(m, n)\right)$. By differentiation of $1+K(s)=K_{+}(s) K_{-}(s)$ we have $K^{\prime}(\alpha)=K_{+}^{\prime}(\alpha) K_{-}(\alpha)$ at a zero $\alpha$ of $K_{+}(s)$ and $K^{\prime}(\beta)=K_{+}(\beta) K_{-}^{\prime}(\beta)$ at a zero $\beta$ of $K_{-}(s)$. Also $K(\alpha)=K(\beta)=-1$. Thus if we omit the inner summations from (7.11), (7.12), each of them is a residue equivalent of

$$
x_{m} \sim-\frac{1}{2 \pi i m} \int_{L} \frac{K(s)}{1+K(s)}\left(\frac{m}{\nu}\right)^{s} d s,
$$

where $L$ is a path $\sigma=$ const. which separates the $\alpha$ on its left from the $\beta$ on its right. Here it is to be noted (i) that the numerator factor $K(s)$, equal to -1 at $\alpha, \beta$, has been chosen so that by (1.7) the integral may converge exponentially, and (ii) that the integrand is regular at the poles of $K(s)$.

Neither of the formulae which thus lead to (7.17) is 'valid' for $m$ near $v$, but now we can add $\delta_{m v}$ to the right of (7.17) and thus obtain a sensible form from which to start ab initio a residual calculation.

We reap the best dividend from these ideas by applying them to the adjoint system. In (3.7) we put

$$
y_{m}=y_{\rho m}=-\frac{1}{2 \pi i \rho} \int_{L^{\prime}} \frac{K(s)}{1+K(s)}\left(\frac{\rho}{m}\right)^{s} d s+\delta_{\rho m}
$$

(the form adjoint to (7.17)) and calculate

$$
h_{\rho n}=y_{\rho n}+\sum_{m} y_{\rho m} k(m, n), \quad \rho=N, N+1, \cdots
$$

Then any solution $\left\{x_{n}\right\}$ of (1.1) which satisfies convergence conditions of the form (3.8), (3.9) must satisfy the equation (cf. (3.6))

$$
\sum_{n} h_{\rho n} x_{n}=\sum_{m} y_{\rho m} g_{m}
$$

From (1.4) and (7.18) we have

$$
\sum_{m} y_{\rho m} k(m, n)=-\frac{1}{2 \pi i} \int_{L^{\prime}} \frac{K(s)}{1+K(s)} \rho^{s-1} d s \int_{L} \frac{K(u) n^{-u} \sum m^{u-1-s} d u}{2 \pi i}+k(\rho, n)
$$

provided $L$ is a path to the left of $L^{\prime}$ in the zero-free strip of $1+K(s)$ containing $L^{\prime}$ (so that $\sum m^{u-1-s}$ may converge absolutely). Now let $L$ be displaced to the right across $L^{\prime}$ to a position $L^{\prime \prime}$ in the strip. The residue of the inner integral at $u=s$ gives to the outer integral the contribution

$$
-\frac{1}{2 \pi i} \int_{L^{\prime}} \frac{K^{2}(s) p^{s-1} n^{-s} d s}{1+K(s)}
$$


and the addition of $y_{\rho n}$ to this gives in all

$$
-\frac{1}{2 \pi i} \int_{L^{\prime}} K(s) \rho^{s-1} n^{-s} d s+\delta_{\rho n}, \quad=-k(\rho, n)+\delta_{\rho n}
$$

by (1.4). Hence (7.19) gives

$$
h_{\rho n}=\delta_{\rho n}-\frac{1}{2 \pi i} \int_{L^{\prime}} \frac{K(s) \rho^{s-1} d s}{1+K(s)} \int_{L^{\prime \prime}} \frac{K(u) n^{-u} \zeta_{N}(1+s-u) d u}{2 \pi i}
$$

When $\rho, n$ are both large we can convert (7.21) into an asymptotic series by displacing $L^{\prime}$ to the left across zeros $\alpha$ of $I+K(s)$ and $L^{\prime \prime}$ to the right across poles $u=q=1,2, \cdots$ of $K(u)$ :

$$
h_{\rho n} \sim \delta_{\rho n}-\sum_{\alpha} \sum_{q} \frac{\zeta_{N}(1+\alpha-q) \text { res. }_{q} K(u) \rho^{\alpha-1} n^{-q}}{K^{\prime}(\alpha)}
$$

where the argument of $\zeta_{N}$ in each term has its real part less than 1 . The leading term has $q=1$ and $\alpha$ with a real part $\alpha_{1}$ less than 1 , and on this account the reduced system (7.20) is regular; indeed, the transformation $x_{n}=n^{\delta} \xi_{n}$ gives a system

$$
\xi_{\rho}+\sum_{n} h_{\rho n}^{\prime} \xi_{n}=\rho^{-\delta} \sum y_{\rho m} g_{m}
$$

in which $h_{\rho n}^{\prime}=O\left(\rho^{-1+\alpha_{1}-\delta} n^{-1+\delta}\right)$, so $\sum \sum\left|h_{\rho n}^{\prime}\right|^{2}$ converges for any $\delta$ in $\alpha_{1}-\frac{1}{2}<\delta<\frac{1}{2}$, a restriction that can be met because $\alpha_{1}<1$.

It may be noted that when only one of $\rho, n$ is large the leading term in (7.22) gives the order of magnitude of $h_{\rho n}$.

The logic of these calculations is as follows. To attain (7.20) we have supposed that the $\left\{x_{n}\right\}$ in question satisfies (1.1), so we make the hypothesis that $\left\{g_{n}\right\}$ is in $D_{\alpha_{2}}$ and (if the index of the strip that contains $L^{\prime}$ is negative) satisfies the consistency condition for solubility of (1.1). Then $\left\{x_{n}\right\}$ is in $D_{\alpha_{1}}$. Also, by Lemma 1 applied to (7.18), $\sum y_{\rho m} g_{m}$ converges, and equals

$$
\sum y_{\rho m} g_{m}=g_{\rho}-\frac{1}{2 \pi i} \int_{L} \frac{K(s) \rho^{s-1}}{1+K(s)} \sum g_{m} m^{-s} d s=G_{\rho}
$$

and similarly $\sum y_{\rho m} x_{m}$ converges. Also by (7.18) $\sum y_{\rho m} m^{c-1}$ converges absolutely to

$$
\rho^{c-1}-\frac{1}{2 \pi i} \int_{L^{\prime}} \frac{K(s) \rho^{s-1}}{1+K(s)} \zeta_{N}(1+s-c) d s
$$

provided $c<\sigma\left(L^{\prime}\right)$, so for fixed $\rho,\left(y_{\rho m}\right)$ is in $D_{1-\beta}$ for any $\beta$ to the right of $L^{\prime}$. Finally (7.21) gives for $\sum h_{\rho n} n^{z-1}$ a form like (7.21) with $n^{-4}$ replaced by $\zeta_{N}(1+u-z)$, so this function is everywhere continuable and at most 
algebraically large for $|\operatorname{Im} z| \sim \infty$. Hence all the hypotheses of Lemma 3 are satisfied, and (7.20) is established. To sum up,

THEOREM 4. If $\alpha$ is to the left of a zero-free strip of regularity of $1+K(s)$ which contains the paths $L^{\prime}$ and $L^{\prime \prime}$ (to the right of $\left.L^{\prime}\right)$, and if $\left\{g_{n}\right\}$ is in $D_{\alpha}$, then any solution $\left\{x_{n}\right\}$ of (1.1) which is in $D_{\alpha}$ satisfies the 'Fredholm' equation

$$
x_{\rho}-\sum_{n} \frac{x_{n}}{2 \pi i} \int_{L^{\prime}} \frac{K(s) \rho^{s-1} d s}{1+K(s)} \int_{L^{\prime \prime}} \frac{K(u) \zeta_{N}(1+s-u) n^{-u} d u}{2 \pi i}=G_{\rho}, \quad \rho \geqq N
$$

where $G_{\rho}$ is defined by (7.24).

Here if the index of the strip is positive there is no restriction of $\left\{x_{n}\right\}$ to any one of the solutions, and the implication is that in this case (7.25) must present the eigen-alternative. There is the correlative circumstance that there is no left-inverse for (1.1) which is associated with the strip in question, so we cannot expect the 'set of multipliers' (7.18) to yield a reduced system that is close to an explicit solution for $\left\{x_{p}\right\}$.

For the calculation of the $x_{n}$ we would usually use (7.25) only for the larger values of $\rho$, say $\rho \geqq N^{\prime}$, whereby $x_{N^{\prime}}, x_{N^{\prime}+1}, \cdots$ are determined in terms of $x_{N}, \cdots x_{N^{\prime}-1}$ as parameters; it is easily proved that this may be achieved by the obvious iterative procedure. The higher coefficients on the left are approximated by (7.22), and for $G_{\rho}$ there is the asymptotic equivalent of (7.24) got by displacing the path to the left, if $\sum g_{m} m^{-8}$ is continuable. The outstanding coefficients, for which $\rho$ but not $n$ is large, may be obtained arithmetically from (7.19). This requires the evaluation of the $y_{\rho m}$ from (7.18), for which asymptotic formulae, obtained by displacing the path to the right or left, are valid except for $\rho$ near $m$; in this outstanding case numerical integration may be needed, but only the one parameter $\rho / m$ is here involved.

\section{Extensions of the theory}

We shall consider briefly some systems of the form

$$
x_{m}+\sum_{n=N}^{\infty} k(m, n) c_{m n} x_{n}=g_{m}
$$

that are in some sense close to $(1.1), k(m, n)$ being the kernel hitherto considered.

8.1. $c_{m n}=a_{m}$, a bounded sequence. For $f_{N}(s)=\sum x_{n} n^{-s}$ we find as in $\S 1.3$ the integral equation

$$
f_{N}(u)+\frac{1}{2 \pi i} \int_{c-i \infty}^{c+i \infty} K(s) f_{N}(s) \sum_{N}^{\infty} a_{m} m^{s-1-u} d s=\sum g_{m} m^{-u},
$$


for $\operatorname{Re} u>c$. The behaviour of this equation depends on the behaviour of the Dirichlet series $\phi(z)=\sum a_{m} m^{-1-z}$ for analytic continuation across the line $\operatorname{Re} z=0$. For example
(i) $a_{m}=1-(-1)^{m}$
gives $\phi(z)=\left(2-2^{-z}\right) \zeta(1+z)$
(ii) $a_{m}=(-1)^{m+1}$
gives $\phi(z)=\left(1-2^{-z}\right) \zeta(1+z)$
(iii) $a_{m}=(-1)^{m+1}+1 / m$
gives $\phi(z)=\left(1-2^{-z}\right) \zeta(1+z)+\zeta(2+z)$.

For (i) we have a pole at $z=0$ with residue 1 , (ii) is everywhere regular, and (iii) has a pole at $z=-1$. In case (i) the equation is singular and the theory and conclusions are like those of $\S \S 3-6$, but in cases (ii), (iii) the equation is regular and the Fredholm theory applies to it; when it has a solution,

$$
x_{m}+\frac{1}{2 \pi i} \int_{c-i \infty}^{c+i \infty} K(s) a_{m} m^{s-1} f_{N}(s) d s=g_{m}
$$

gives a corresponding solution of (8.1).

8.2. $c_{m n}=\left(1+a_{m}\right)\left(1+b_{n}\right) ; a_{m}, b_{m}=O\left(m^{-\delta}\right)$ for some positive $\delta$. By substituting for $k(m, n)$ from (1.4) we get a form in which the variables $m, n$ are separated in the integrand, and as in $\S 1.3$ we find the equivalent integral equation

$$
\begin{array}{r}
f_{N}(u)+\frac{1}{2 \pi i} \int_{L} K(s) f_{N}(s) \sum m^{s-u-1}\left(1+a_{m}\right)\left(1+b_{m}\right) d s \\
=\sum g_{m}\left(1+b_{m}\right) m^{-u}=g_{N}(u)
\end{array}
$$

for $f_{N}(s)=\sum x_{n}\left(1+b_{n}\right) n^{-s}$. Thus in place of $\zeta_{N}(z)$ we have $\zeta_{N}(z)+\chi_{N}(z)$, where

$$
\chi_{N}(z)=\sum_{N}^{\infty}\left(a_{m}+b_{m}+a_{m} b_{m}\right) m^{-z},
$$

a Dirichlet series converging absolutely for $\operatorname{Re} z>1-\delta$, so that $\chi_{N}(z)=O\left(N^{1-z-\delta}\right)$ for $\operatorname{Re} z \geqq 1-\frac{1}{2} \delta$. In (4.4) $\eta_{N}(1+u-s)$ is replaced by $\eta_{N}(1+u-s)+\left(N-\frac{1}{2}\right)^{u-s} \chi_{N}(1+u-s)$, and the additional term is $O\left(N^{-8}\right)$ for $\operatorname{Re}(u-s)>-\frac{1}{2} \delta$. The same adjustment applies to (4.14) and the conclusions of $\S \S 3-6$ remain valid, the work requiring only trivial modifications.

The continuation of $f_{N}(u)$ to the left of the path $L$ in (8.3), such as would be required for work like that of $\S 7$, is interesting. It is given by converting (8.3) to

$$
(1+K(u)) f_{N}(u)+\frac{1}{2 \pi i} \int_{L^{\prime}} K(s) f_{N}(s)\left(\zeta_{N}(1+u-s)+\chi_{N}(1+u-s)\right) d s=g_{N}(u)
$$

with $L^{\prime}$ just to the right of $L$. In the first instance $u$ is restricted to the strip 
of breadth $\delta$ to the left of $L^{\prime}$ in which $\chi_{N}$ is regular, and $f_{N}(u)$ is therein regular, provided $g_{N}(u)$ is so. Hence we can displace the path in (8.4) to the left through a distance $<\delta$, and so obtain a further continuation, provided $g_{N}(u)$ permits it. This process continuous until $f_{N}(u)$ acquires a pole at a zero $\alpha$ of $1+K(u)$. But when the path is moved across this pole an additional residue-term carrying the factor $\chi_{N}(1+u-\alpha)$ enters, and the singularities of this function become dominant; for example $\chi_{N}(z)$ may have a natural boundary $\operatorname{Re} z=1-\delta$, and then $f_{N}(u)$ has a corresponding boundary on $\operatorname{Re} u=\operatorname{Re} \alpha-\delta$.

8.3.

$$
c_{m n}=1+d_{m n}, \quad d_{m n}=O\left(m^{-\kappa} n^{-\eta}\right) .
$$

We confine attention mainly to the case where there is a zero-free strip of regularity $\alpha<\sigma<\beta$ of $1+K(s)$ whose index is 0 . The complete system (8.1) can be partitioned as in $\S 6$, so as regards the circumstances that are peculiar to the case (8.5) we can confine attention to the case (§ 6.4) where the matrix

$$
\boldsymbol{M}=\left(\delta_{m n}+k(m, n)\right)
$$

has a left- and right-inverse $M^{-1}$ with columns in $D_{\alpha}$ and rows in $D_{1-\beta}$. Writing $\boldsymbol{D}=\left(k(m, n) d_{m n}\right)$ the system $(8.1)$ is

$$
\boldsymbol{M x}+\boldsymbol{D x}=\boldsymbol{g} .
$$

If convergence conditions are satisfied this implies

$$
\boldsymbol{x}+\boldsymbol{M}^{-1} \boldsymbol{D} \boldsymbol{x}=\boldsymbol{M}^{-1} \boldsymbol{g},
$$

which in turn implies (8.6). Thus (8.7) is equivalent to (8.6), and we are to find conditions on $\kappa, \eta$ under which it is regular.

From (1.4) and (8.5) we have, for the typical element of $D$,

$$
k(m, n) d_{m n}=O\left(m^{0-1-\kappa} n^{-c-\eta}\right)
$$

for any $c$ for which (1.4) is applicable: in general $0<c<1$, or in the case of $\S 2.1-l<c<j+1$. As regards $M^{-1}$ the typical element $y_{\rho m}$ is given asymptotically by (7.18), so for all $\rho, m$ we have

$$
y_{\rho m}=\delta_{\rho m}+O\left(\rho^{\mathrm{c}^{\prime}-1} m^{-c^{\prime}}\right)
$$

for any $c^{\prime}$ between $\alpha$ and $\beta$. For $\boldsymbol{M}^{-1} \boldsymbol{D}$ the typical element is then

$$
\begin{aligned}
h_{\rho n}=\sum_{m} y_{\rho m} k(m, n) d_{m n} & =k(\rho, n) d_{\rho n}+\sum_{m} O\left(\rho^{c^{\prime}-1} m^{c-1-\kappa-c^{\prime}} n^{-c-\eta}\right) \\
& =O\left(\rho^{c-1-\kappa} n^{-c-\eta}\right)+O\left(\rho^{c^{\prime}-1} n^{-c-\eta}\right)
\end{aligned}
$$

provided

$$
c^{\prime}-c+\kappa>0,
$$


so that the $m$-summation may converge. Then the last term in (8.9) is dominant, and if the sum of its indices $<-1$ the reduced system (8.7) is regular. Hence we require

$$
-\kappa<c^{\prime}-c<\eta \text {, }
$$

which is to be satisfiable by a choice of $c^{\prime}$ between $\alpha, \beta$ and a choice of $c$ between $-l$ and $j+1$; and this is so if

$$
\kappa+\eta>0, \quad \kappa>-\beta-l, \quad \eta>\alpha-1-j .
$$

Under these conditions the Hilbert theory, including the Fredholm Alternative theorems, is applicable to (8.7). Its solubility will of course depend on $\boldsymbol{M}^{-1} \boldsymbol{g}$ belonging to a suitable sequence-space. For example, if $g_{m}=O\left(m^{\alpha-1}\right)$, the typical element $\sum_{m} y_{\rho m} g_{m}$ of $\boldsymbol{M}^{-1} \boldsymbol{g}$ is $O\left(\rho^{c^{\prime}-1}\right)$, by (8.8), where $c^{\prime}>\alpha$. By (8.9) and (8.11), then,

$$
\sum_{n=N^{\prime}}^{\infty} h_{\rho n} O\left(n^{c^{\prime}-1}\right)
$$

converges and is $o\left(\rho^{c^{\prime}-1}\right)$ for $N^{\prime} \sim \infty$; so if we omit from (8.7) a finite set of the early equations, the remaining infinite system is soluble by a diagonalbased iteration.

An alternative and more practical procedure for reducing the system (8.6) is to left-multiply it by the approximation to $\boldsymbol{M}^{-1}$ given by (7.18); the typical coefficient in the reduced system then contains an extra component as shown on the right of (7.21). Indeed, we can use (7.18) as a set of multipliers irrespective of the index of the strip $\alpha<\sigma<\beta$ with which they are associated, and the reduced system is regular under the conditions (8.12); but the equivalence of the reduced and original systems is not thereby established.

\section{References}

[1] E. Hellinger and $O$. Toeplitz, Integralgleichungen und Gleichungen mit unendlich vielen Unbekannten. Leipzig \& Berlin, Teubner, 1928. Nr. 16.

[2] A. Erdélyi et al., Tables of Integral Transforms Vol. I, Bateman Manuscript Project. New York, McGraw Hill, 1954.

[3] N. I. Muskhelishvili, Singular Integral Equations (Transl. by J. R. M. Radok) Groningen, Noordhoff, 1953.

[4] G. H. Hardy and M. Riesz, The General Theory of Dirichlet's Series. Cambridge Tracts in Mathematics and Mathematical Physics. C.U.P., 1915.

[5] A. C. Dixon, Proc. London Math. Soc. [2] 27, 233-272 (1928).

[6] E. C. Titchmarsh, Introduction to the Theory of Fourier Integrals, Oxford, Clarendon Press, 1937, Sec. 11.18

[7] G. H. Hardy and J. E. Littlewood, Proc. London Math. Soc. [2] 21, 39-74 (1922).

[8] See Nr. 18 of Reference 1.

Department of Mathematics, University of Melbourne. 